\title{
Partial wave analysis
}

\author{
Eberhard Klempt ${ }^{1, \star}$, Andrey V. Sarantsev ${ }^{1,2, \star \star}$, and Ulrike Thoma ${ }^{1, \star \star \star}$ \\ ${ }^{1}$ Helmholtz-Institut für Strahlen- und Kernphysik, Universität Bonn, 53115 Bonn, Germany \\ ${ }^{2}$ National Research Centre "Kurchatov Institute", Petersburg Nuclear Physics Institute, Gatchina, Russia
}

\begin{abstract}
The project A.2 of the SFB/TR16 "Subnuclear Structure of Matter" aimed at a combined multichannel partial wave analysis of all or of most data on pion and photo-induced reactions for invariant masses from the $\Delta(1232)$ region to $2300 \mathrm{MeV}$. In this review the most important results are presented. It is shown that the data and their partial wave analysis have improved our understanding of the dynamics of excited light-quark baryons significantly.
\end{abstract}

\section{Introduction}

"Why baryons?" asked Nathan Isgur [1] at NSTAR2000, and gave three answers: Because nucleons are the stuff of which our world is made, because they are the simplest system in which the non-abelian character of QCD is manifest, and because their complexity - compared to mesons - may reveal physics hidden from us in mesons. And he predicted that baryon spectroscopy will be one of the most interesting and fruitful areas for at least thirty years. Recent reviews of baryon spectroscopy by Klempt and Richard [2] and Crede and Roberts [3] underline the interest in the field.

Isgur's high expectations were based on severe discrepancies between the experimental findings and the spectrum of nucleon excitations predicted in quark models (e.g. [4-9]) or in recent calculations within lattice QCD [10] (even though these calculations still use quark masses corresponding to a large pion mass, $\mathrm{m}_{\pi}=396 \mathrm{MeV}$ ).

In 2000, the knowledge of the excitation spectrum of the nucleon stemmed essentially from data on $\pi N$ elastic scattering [11-13]. In quark models, the predicted spectrum is organized in excitation shells, and a shell structure is clearly seen in the experimental spectrum. However, the resonance spectrum which emerged exhibited two severe problems:

A) The number of states expected for a system with three constituent quarks exceeded by far the number of observed states.

The authors of, e.g., ref. [6] list $66 N^{*}$ and $35 \Delta^{*}$ resonances predicted to have masses below $2.5 \mathrm{GeV}$. However, the Review of Particle Properties of 2000 [14] quotes only $19 N^{*}$ and $19 \Delta^{*}$ in this mass range. And, even worse,

\footnotetext{
$\star$ e-mail: klempt@hiskp.uni-bonn.de

$\star \star$ e-mail: andsar@hiskp.uni-bonn.de

$\star \star \star$ e-mail: thoma@hiskp.uni-bonn.de
}

Arndt and his collaborators [15] found only $8 \mathrm{~N}^{*}$ and 10 $\Delta^{*}$-states. This latter analysis included high precision data from the meson factories at LAMPF, PSI, and TRIUMF. A strong argument in favor of the Arndt et al. analysis was the correct prediction of spin rotation parameters [16-18] and of the backward asymmetry [19] in the elastic pionproton scattering from ITEP/PNPI; the predictions from $[11,12]$ show clear discrepancies with the data.

Clearly, not all 101 resonances expected below $2500 \mathrm{MeV}$ need to be found to reach a good unterstanding of the spectrum. In the first excitation shell 5 negativeparity $N^{*}$ 's and $2 \Delta^{*}$ 's are predicted and experimentally established. In the second shell, 14 positive-parity $N^{*}$ 's and $8 \Delta^{*}$ 's are predicted; their calculated masses fall below $2100 \mathrm{MeV}$. Based on [11, 12] (or [15]), only 8 (or 4) positive-parity $N^{*}$ 's and 6 (or 5) $\Delta^{*}$ 's are actually observed. Only 4 (instead of 14 ) positive-parity $N^{*}$ 's are safe: this small number casts into doubt if models based on constituent quarks with some residual interactions are the right degrees of freedom to describe the nucleon excitation spectrum.

The problem of missing baryon resonances is aggravated by the prediction of additional states, hybrid baryons, in which the gluonic string mediating the interaction between the quarks is itself excited. Hybrid baryons carry the same quantum numbers as $q q q$ baryons, $q q q$ and hybrid configurations can mix and are thus difficult to establish. In a quark model [20], the baryon hybrid spectrum (with no mixing with $q q q$ baryons) is calculated to intrude the spectrum of baryon resonances at $2 \mathrm{GeV}$ and above. In lattice calculations [21], most baryons with a dominant hybrid content show up above $2500 \mathrm{MeV}$, except for $J^{P}=1 / 2^{+}$where $N(1440) 1 / 2^{+}$and $N(1710) 1 / 2^{+}$are predicted to acquire a significant hybrid component.

A well-known solution of the problem of the missing resonances is the conjecture that baryon excitations could be driven by the dynamics of a quark and a (quasi-stable) diquark. There is a long discussion on the nature and rel- 
evance of the diquark concept (see e.g. [22-25]). This hypothesis decreases the number of expected resonances very significantly and helps to solve the problem of the missing baryon resonances. Santopinto, e.g., calculated the $N^{*}$ and $\Delta^{*}$ excitation spectrum [26] with the assumption that the baryon is made up from a point-like diquark and a quark. The results match data perfectly, provided $N^{*}$ - and $\Delta^{*}$-resonances are omitted that had an one- or twostar ranking only in PDG2004 [27].

Alternatively, the missing resonances may have escaped detection due to their small coupling to the $\pi N$ channel [28]. The question arizes: are there baryon resonances with small $\pi N$ couplings which can be produced by electromagnetic excitation and which decay into other final states?

A further point of concern is the observation that

B) the pattern of observed states is neither compatible with quark model calculations nor with present lattice calculations.

In particular the low mass of the Roper resonance $N(1440) 1 / 2^{+}$has led to vivid discussions in the literature. Inconsistencies in different data sets led Morsch and Zupranski [29] to conjecture that the $1400 \mathrm{MeV}$ region might even house two resonances, a hypothesis which we ruled out in [30]. Most papers on the Roper resonance address the question if it is a "normal" qqq resonance, a low-mass hybrid baryon [31, 32], or if it is dynamically generated from meson-baryon interactions [33]. If the Roper resonance would be exotic, $N(1710) 1 / 2^{+}$would be the true first radial excitation of the nucleon. However, electroexcitation of the Roper resonance provides strong evidence for this state as a predominantly radial excitation of a 3-quark ground state [34]. Note, that any exotic interpretation of the Roper resonance should explain the low masses of the corresponding states in the $\Lambda, \Sigma$, and $\Xi$ spectra. Likewise, the $\Lambda(1405) 1 / 2^{-}$resonance has attracted intense interest since Dalitz proposed it to be generated dynamically [35]. This topic is treated in project B.3.

The generation of baryon resonances from the interaction of mesons and baryons is a highly attractive approach to understand the dynamics of baryon resonances. At present, however, the number of expected resonances is unknown, and the relation between quark model states and dynamically generated resonances is not understood. It is hence important to measure the decay modes of all major decay modes.

At higher energies, the masses of $\Delta(1900) 1 / 2^{-}$, $\Delta(1940) 3 / 2^{-}, \Delta(1930) 5 / 2^{-}$are incompatible with quark model predictions (e.g. [6]). They belong to the third excitation shell, and their masses should fall into the $2100-2300 \mathrm{MeV}$ range. Instead, they are approximately mass degenerate with the positive-parity $\Delta(1910) 1 / 2^{+}$, $\Delta(1920) 3 / 2^{+}, \Delta(1905) 5 / 2^{+}, \Delta(1950) 5 / 2^{+}$. Such parity doublets occur frequently in hadron spectra [36]; Fig. 1 shows parity doublets in the $N^{*}$ spectrum. Parity doublets are observed undoubtedly. Similar observations in the meson [37] and baryon [38] sector have led to the idea that chiral symmetry might be effectively restored in highly

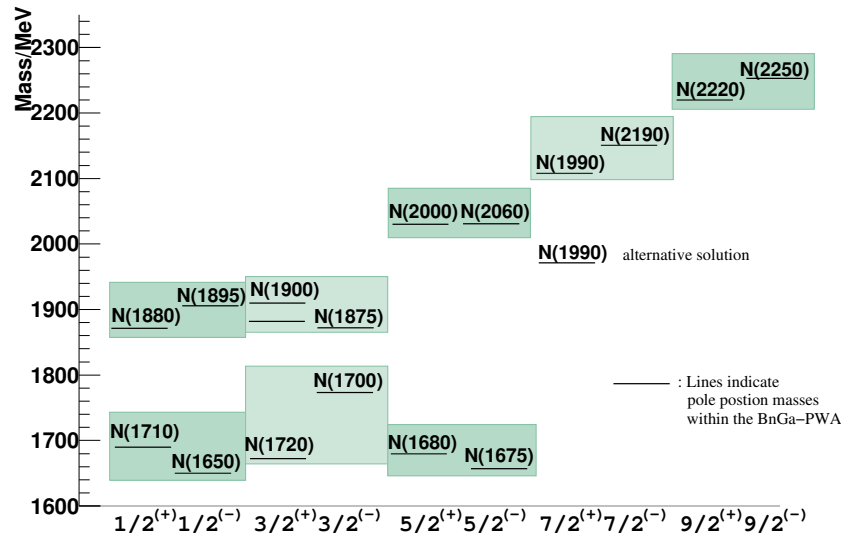

Figure 1. Examples of nucleon resonances organized as parity doublets. The lines indicate the masses of $\mathrm{BnGa}$ pole position of the respective states. For the $7 / 2^{+}$-state two alternative solutions exist: one close to the $N(2190) 7 / 2^{-}$, the other one at a lower mass, both are shown. The pole masses of $N(1720) 3 / 2^{+}$and $N(1700) 3 / 2^{-}$are found $110 \mathrm{MeV}$ apart, all other parity doublets indicated have a significantly smaller mass splitting.

excited hadrons. If this would indeed be the case chiral partners were expected to exist for all high-mass states. Independently of the idea of chiral symmetry restoration, the question whether or not parity partners exist for all highmass states is an interesting question to be answered experimentally.

Photoproduction of baryon resonances is hence studied to find answers to the following questions:

1. Is the number of observed resonances compatible with diquark models?

2. Is their evidence for three-quark dynamics in excited baryon states?

3. What is the dynamical reason for the existence of parity doublets?

4. Which resonances are seen in the third excitation shell?

5. Can baryon resonances be assigned to specific $S U(6) \otimes O(3)$ multiplets?

Based on the new data taken during the SFB/TR16 funding period, further questions arose:

6. Is photoproduction of $\omega$ mesons governed by diffraction, or play resonances a significant role?

7. Is the narrow peak seen in the reaction $\gamma n \rightarrow \eta n$ a resonance?

8. What is the relation between quark-model resonances and dynamically generated resonances?

9. Do the new photoproduction data with polarized photon beams and polarized targets lead to consistent multipoles when analyzed with different PWA methods?

10. Can photoproduction multipoles be determined in slices of fixed masses?

Photoproduction data were taken within this SFB/TR16 (Projects A.1 "Spectroscopy of baryon resonances", B.1 "Protoproduction of mesons"). These data and data from 
other laboratories provide important information used in the comprehensive analysis of photo- and pion-induced reactions performed within the Bonn-Gatchina partial wave analysis (BnGa-PWA) (project A.2 within the SFB/TR16) presented here. The importance of the recent single and double polarization observables becoming available from ELSA, JLab, and MAMI cannot be overestimated. They provide very important constraints for the PWA.

\section{The Bonn-Gatchina PWA, data base and method}

As composite systems, nucleons exhibit a rich spectrum of radial and orbital excitations. The excitation levels of the nucleon are extremely short-lived and decay in a variety of different decay modes. Many states are predicted which overlap and are very difficult to resolve. Hence the aim to identify all $N^{*}$ and $\Delta^{*}$ resonances contributing to photoproduction and to measure all important decay modes requires a simultaneous fit to a large body of data in a multi-channel partial wave analysis. The following data were included in the analysis:

1. Data on the photoproduction off protons and off neutrons obtained at ELSA and at other laboratories. This includes data on single as well as on double meson photoproduction. The latter data are fitted in terms of an event-based maximum likelihood fit to take all the correlations between the variables the event depends on properly into account.

2. Real and imaginary part of the partial wave amplitudes for $\pi N$ elastic scattering either from Höhler's group or from Arndt's group.

3. Pion-induced inelastic reactions.

The latter data (2) and (3) ensure consistency with the $\pi N$ scattering data and allow the extraction of the $\pi N$ coupling strength of baryon resonances. Fits to photoproduction data alone would provide only access to the product of the photon helicity amplitudes $A_{1 / 2}, A_{3 / 2}$ and the square root of the decay branching ratio into the specific final state, $A_{1 / 2 \text { or } 3 / 2} \cdot \sqrt{B R_{\text {final } i}}$ with $B R_{\text {final } i}=\Gamma_{\text {final } i} / \Gamma_{\text {tot }}$ (with $\Gamma_{\text {final } i}$ being the partial decay width into the final state $\mathrm{i}$ and $\Gamma_{t o t}$ the total width of the resonance).

The following reactions are presently included in the BnGa-PWA. (Most data can be found via the web page of the GWU PWA group [39]):

\begin{tabular}{l|l}
\hline \hline Photoproduction & $\pi N$-scattering \\
\hline$\gamma p \rightarrow \pi^{0} p, \pi^{+} n$ & $\pi^{-} p \rightarrow \pi^{-} p$ (elastic)-partial waves \\
$\gamma p \rightarrow \eta p, \eta^{\prime} p$ & $\pi^{-} p \rightarrow \eta n$ \\
$\gamma p \rightarrow \omega p, K^{*+} \Lambda$ & \\
$\gamma p \rightarrow K^{+} \Lambda$ & $\pi^{-} p \rightarrow K^{0} \Lambda, K^{+} \Sigma^{-}, K^{0} \Sigma^{0}, K^{-} \Sigma^{+}$ \\
$\gamma p \rightarrow K^{+/ 0} \Sigma^{0 /+}$ & $\pi^{+} p \rightarrow K^{+} \Sigma^{+}$ \\
$\gamma p \rightarrow p \pi^{0} \pi^{0}, p \pi^{+} \pi^{-}$ & $\pi^{-} p \rightarrow n \pi^{0} \pi^{0}, n \pi^{+} \pi^{-}$ \\
$\gamma p \rightarrow p \pi^{0} \eta$ & \\
$\gamma n \rightarrow \pi^{0} n, \pi^{-} p$ & \\
$\gamma n \rightarrow \eta n$ & \\
\hline \hline
\end{tabular}

This data base has been extended continuously over the last 12 years. In particular the new polarization data were included in the fits whenever they became available. Very recently also (so far unpublished) data on photoproduction of two charged pions (CLAS) were added.

A multi-channel analysis of a large body of data has a large advantage; in contrast to an analysis of $\pi N \rightarrow \pi N$ elastic scattering alone, the simultaneously fitted photoproduction data provide access to resonances which might have escaped detection in $\pi N$ scattering due to their insufficient $\pi N$-coupling. At the same time the photoproduction data constrain the elastic amplitudes by fixing the structure of the otherwise unknown inelasticities. Polarization observables measured in photoproduction reactions provide sensitive information on the interferences of different partial waves. This helps to extract even small resonance contributions from the data. Simultaneous fits to different single and double polarization observables exploit different interference effects and are hence very sensitive to small amplitudes.

The fit method has been documented in a series of papers [40-43]. Here we just mention that the amplitudes for pion- and photo-induced reactions are represented by a sum of K-matrix partial wave amplitudes and by background amplitudes describing $u$ - and $t$-channel exchanges. The corresponding exchange amplitudes are written in the form of the exchange of Regge trajectories. The K-matrix amplitudes contain poles and simple functions of $s$ to model additional background. Methods for the decomposition of the background amplitudes from $t$ - and $u$-channel exchanges into partial waves have been developed and implemented. These allow the direct comparison of our partial waves (or multipoles) with those of other analyses as discussed, e.g., in section 3.9.

Due to the so far still incomplete data base, different solutions are found which describe the data with similar quality. We always quote errors which cover all solutions or we discuss different classes of solutions in which the number and the properties of some resonances may differ [44-49].

It should also be mentioned, that within the $\mathrm{BnGa}$ approach, we do not attempt to provide a microscopical description of the background starting from a Lagrangian like it is done by other groups like Argonne-Osaka [50], Jülich-Bonn [51-56], Dubna-Mainz-Taipeh [57], EBACJlab [58], Valencia [59], or within the Gießen Coupled Channel analysis $[60,61]$ (see also contribution of project B.7 "Coupled channels approach to photo-meson production on the nucleon" to these proceedings). These models have the advantage that the number of free parameters for the background is minimized while problems might occur if the dynamical model is incomplete and the fit is constrained to it.

A purely phenomenological model as the BnGa-PWA satisfying basic requirements is hence a meaningful tool to access properties of resonances. The method employed here satisfies unitarity and the amplitudes are analytic functions in the complex energy plane. The method has recently been improved by introducing a $N / D$-based method 
in our fitting [62]. Studies on baryons indicate that the effects on the amplitudes are mostly small, and that changes in the resonance parameters are covered by the errors we have quoted in our analyses based on the K-matrix formalism.

\section{Results}

\subsection{Can we rule out diquark models?}

The Particle Data Group (PDG) summarizes biannually the status of particle physics. Their Review of Particle Physics (RPP) contains a listing of known baryon resonances and their properties. Before the 2007 issue, the information on nucleon and $\Delta$ excitations came nearly entirely from the groups led by Höhler [11], Cutkosky [12], and Arndt [15] analyzing data on $\pi N$ elastic and charge exchange scattering, with a few additions from Manley [63]. However, the GWU group reduced continuously the number of observed resonances required in their fits $[13,15,64]$. Before 2006, data on photoproduction added information on the helicity couplings of resonances listed in the RPP, but the resonances were introduced into the respective analyses with fixed masses and widths. Early results of the $\mathrm{BnGa}$ group using data on photoproduction from the CB-ELSA experiment [65-68] were not yet included in the RPP $[44,69]$ or not yet used to define particle properties [30, 70]

The BnGa group was the first one which combined pion and photoproduced reactions in a coupled channel fit, fitted masses and widths freely and searched systematically for new resonances [46-49]. In this way, the baryon resonances dismissed in the Arndt et al. analysis [15] could be re-established [46]. In [49], we have reported the observation of $21 \mathrm{~N}^{*}$ and of $10 \Delta^{*}$ resonances and determined their masses, width, and a number of decay modes. Six nucleon resonances were new in the sense that they had no own PDG entry before (even though earlier experiments may also have found evidence for their existence). These results entered the RPP2012 [71].

Table 1 lists the resonances found in the $\mathrm{BnGa}$ analysis which were new or for which the star rating was increased.

Table 1. New states found by the BnGa PWA, which have been introduced in the new PDG'2012, or states in which the BnGa PWA improved the star rating of the resonance. "no evidence" in the GWU Arndt'06 analysis indicates states given in PDG'2010

which were not confirmed in the GWU Arndt'06-analysis.

\begin{tabular}{|l|c|c|c|c|}
\hline & $\begin{array}{c}\text { RPP } \\
2010\end{array}$ & $\begin{array}{c}\text { our } \\
\text { analyses }\end{array}$ & $\begin{array}{c}\text { RPP } \\
2012\end{array}$ & GWU'06 \\
\hline $\mathrm{N}(1860) 5 / 2^{+}$ & & $*$ & $* *$ & \\
$\mathrm{~N}(1875) 3 / 2^{-}$ & & $* * *$ & $* * *$ & \\
$\mathrm{~N}(1880) 1 / 2^{+}$ & & $* *$ & $* *$ & \\
$\mathrm{~N}(1895) 1 / 2^{-}$ & & $* *$ & $* *$ & \\
$\mathrm{~N}(1900) 3 / 2^{+}$ & $* *$ & $* * *$ & $* * *$ & no evidence \\
$\mathrm{N}(2060) 5 / 2^{-}$ & & $* * *$ & $* *$ & \\
$\mathrm{~N}(2150) 3 / 2^{-}$ & & $* *$ & $* *$ & \\
$\Delta(1940) 3 / 2^{-}$ & $*$ & $* *$ & $* *$ & no evidence \\
\hline
\end{tabular}

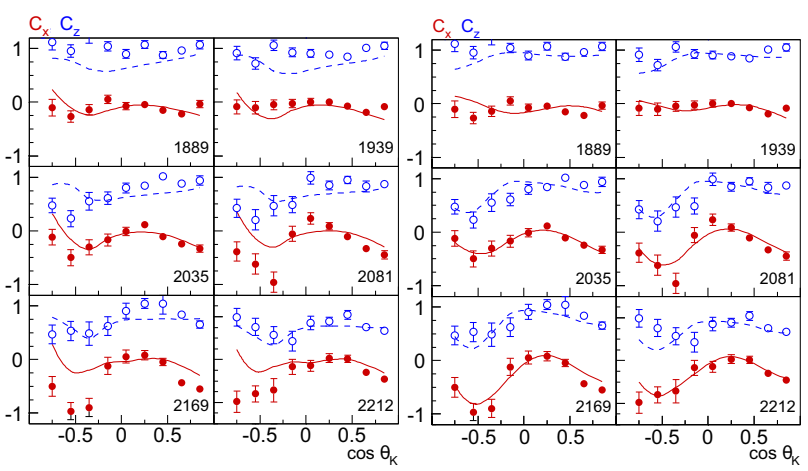

Figure 2. Double polarization observables $C_{x}$ (red points) and $C_{z}$ (open blue circles) for $\gamma \mathrm{p} \rightarrow \mathrm{K}^{+} \Lambda$ [72]. The solid $\left(C_{x}\right)$ and dashed $\left(C_{z}\right)$ curves are our result obtained without (left panel) and with the $N(1900) 3 / 2^{+}$state (right panel) included in the fit.

When comparing RPP'2000 with RPP'2010, we notice that not a single new baryon resonance entered the RPP during these 10 years. With the new high-quality photoproduction data, including single and double polarization observables, the situation has now changed dramatically.

The evidence for one of the states, $N(1900) 3 / 2^{+}$, can be seen in Fig. 2. The figure shows the CLAS beam-recoil polarization data $\left(C_{x}, C_{z}\right)$ for the reaction $\gamma p \rightarrow K^{+} \Lambda$ (selected bins only) [72] in comparison to the best fit obtained without and with $N(1900) 3 / 2^{+}$included in the PWA [73]. The improvement due to the $N(1900) 3 / 2^{+}$is clearly visible.

The number of observed states is now definitely larger than expected from diquark models and supports symmetric quark models in which three constituent quarks participate in the dynamics of excited states.

\subsection{Baryon cascade decays reveal three-quark dynamics}

Three-body decays of baryon resonances give access to sequential decays of baryon resonances via intermediate baryon or meson resonances. The photoproduction reactions $\gamma p \rightarrow \pi^{0} \pi^{0} p$ [74] and $\gamma p \rightarrow \pi^{0} \eta p[75,76]$ were included in the PWA using an event-based maximum likelihood fit to properly consider all the correlations the reaction depends on in the given 5-dimensional phase space. The data were taken at ELSA with the CBELSA/TAPS experiment within project A.1. Data from GRAAL [77] and MAMI [78] on $\gamma p \rightarrow \pi^{0} \pi^{0} p$ were included in the analysis, the MAMI data on $\gamma p \rightarrow \pi^{0} \eta p$ [79] were added to the data base later.

A first example can be seen in Fig. 3. In the reaction $\gamma p \rightarrow \pi^{0} \eta p$ and for a mass range of $2000 \pm 100 \mathrm{MeV}$ masses, $\Delta^{*}$ decays into $\Delta(1232) \eta$ are observed as well as $N^{*}$ or $\Delta^{*}$ decays into $N a_{0}(980)$. Contributions from the isobar $N(1535) 1 / 2^{-} \pi$ emerge clearly, in particular in the $2200 \pm 100 \mathrm{MeV}$ mass range. A partial wave analysis gives the contributing resonances and their decay frequencies into these isobars [76]. The most significant contribution stems from a $\Delta(1232) \eta$ threshold enhancement and is assigned to $\Delta(1700) 3 / 2^{-}$production. But also $\Delta(1940) 3 / 2^{-}$ decays significantly into the $\Delta(1232) \eta$ isobar: because of 

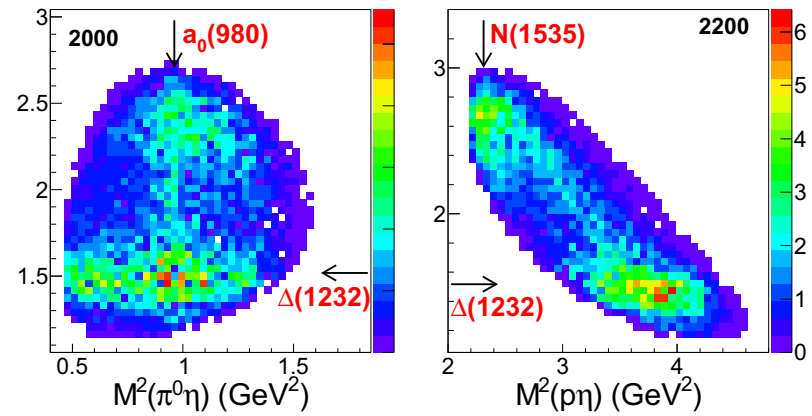

Figure 3. Dalitz plots (taken from [76]) for the reaction $\gamma p \rightarrow$ $\pi^{0} \eta p$ for the mass range $2000 \pm 100 \mathrm{MeV}$ and $2200 \pm 100 \mathrm{MeV}$ (CBELSA/TAPS-data). Evidence is seen for the intermediate states $\Delta(1232) \eta, p a_{0}(980)$, and $N(1535) 1 / 2^{-} \pi$.
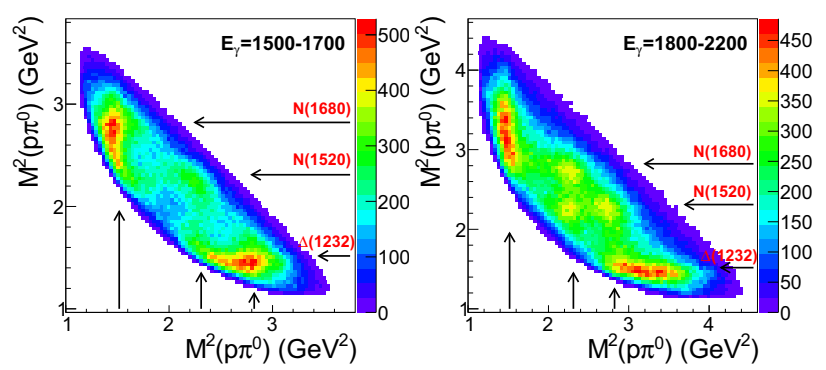

Figure 4. Dalitz plots for the reaction $\gamma p \rightarrow p \pi^{0} \pi^{0}$ (CBELSA/TAPS) [74]. Shown are examples from two energy bins. the pictures are taken from [74], which also provides firther details).

this observation, the PDG upgraded the $\Delta(1940) 3 / 2^{-}$resonance from one star to two stars.

Fig. 4 shows two Dalitz plots on $\gamma p \rightarrow \pi^{0} \pi^{0} p$. Several isobars are seen. Higher-mass baryon resonances seemingly prefer to decay into other high-mass resonances with a low decay momentum, instead of choosing a highmomentum decay into a low-mass meson and a nucleon: Apparently, the intrinsic wave functions of resonances do not contain large components with high momenta. QCD likes to invest into mass and not only into momentum.

The analysis of the data allows us to extract branching ratios for the decays of resonances into $\Delta \pi, \Delta \eta$, $N(\pi \pi)_{S}, \mathrm{~N}(1440) 1 / 2^{+} \pi, \mathrm{N}(1520) 3 / 2^{-} \pi, N(1535) 1 / 2^{-} \pi$, and $N(1680) 5 / 2^{+} \pi$. In total, more than 400 properties of
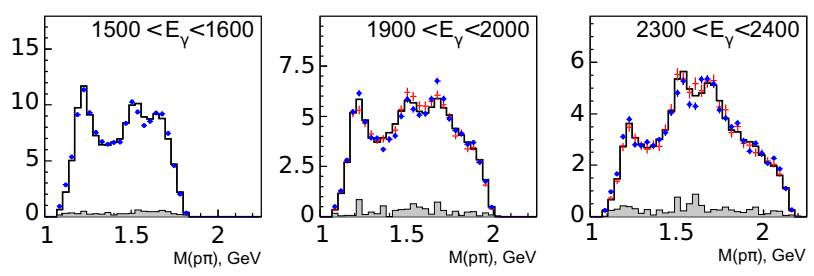

Figure 5. $d \sigma / d M\left(p \pi^{0}\right)$ for $\gamma p \rightarrow p \pi^{0} \pi^{0}$ : examples for three $p \pi^{0}$-invariant mass bins. The blue an red data points show two different CBELSA/TAPS data sets. The systematic uncertainty of the data is shown as grey band. In addition, there is a normalization uncertainty of $10 \%$ [74]. The result of the BnGa-PWA is shown as black curve. baryon resonances were determined, many of them were obtained for the first time. They will be listed in the 2016 edition of the RPP. Fig. 5 shows invariant masses in comparison to the $\mathrm{BnGa}$ fit.

In the $1900 \mathrm{MeV}$ region, for $1300<E_{\gamma}<1650 \mathrm{MeV}$, the cascade $N^{*} \rightarrow N(1520) \pi^{0} \rightarrow p \pi^{0} \pi^{0}$ is dominated by the $J^{P}=3 / 2^{+}$partial wave. This can be seen by plotting the variables $I_{s}, I_{c}$ defined by

$$
\frac{\mathrm{d} \sigma}{\mathrm{d} \Omega}=\left(\frac{\mathrm{d} \sigma}{\mathrm{d} \Omega}\right)_{0}\left\{1+P_{l}\left[I^{s} \sin (2 \phi)+I^{c} \cos (2 \phi)\right]\right\} .
$$

$I_{s}, I_{c}$ carry information on the spin density matrix of the $N(1520) 3 / 2^{-}$isobar. The variables can be plotted as functions of $\phi^{*}[74,80]$ and are shown repeatedly in Fig. 6. Superimposed are two-parameter fits assuming different partial waves in the initial state. The coupling strength and the fractional contribution of the higher partial-wave contribution are varied in the fits. Only one fit gives a reasonable fit to the data; it assumes $J^{P}=3 / 2^{+}$in the initial state. This is an important result. It is shown that in this cascade, one partial wave is dominant. The result is fully model independent and has been achieved without any partial wave analysis just calculating the respective angular distributions!

The decays of the $N^{*}$ and $\Delta^{*}$-resonances reveal evidence for the three-particle dynamics of excited nucleon resonances. $\Delta(1910) 1 / 2^{+}, \Delta(1920) 3 / 2^{+}$, $\Delta(1905) 5 / 2^{+}$, and $\Delta(1950) 7 / 2^{+}$show rather small de-

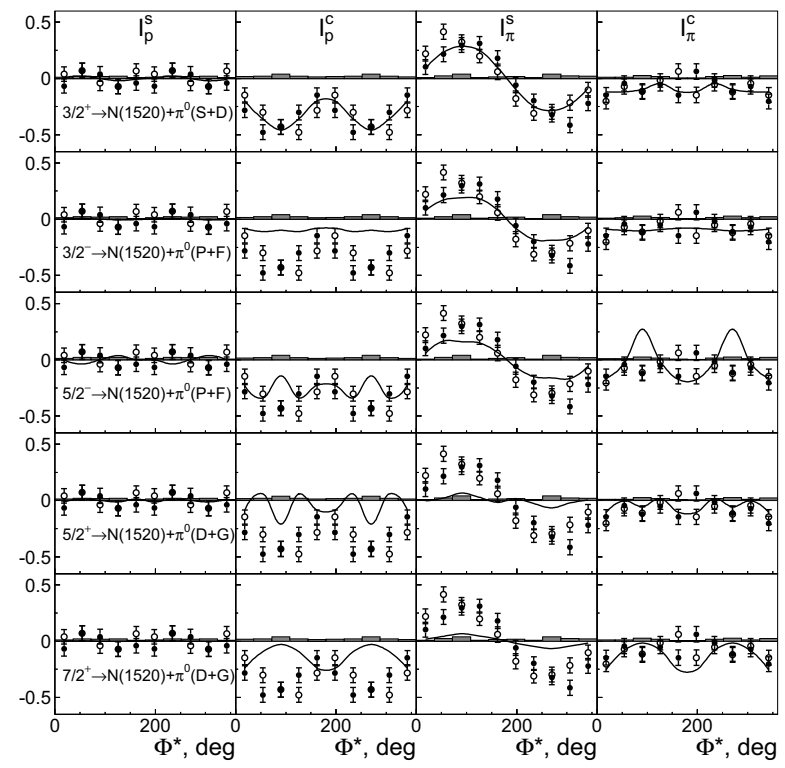

Figure 6. $I^{s}$ and $I^{c}$ for events with a proton $\pi^{0}$ invariant mass above the $\Delta(1232)$ mass for $1300<E_{\gamma}<1650 \mathrm{MeV}$. Solid symbols: extracted directly from the data, open symbols: mirror points due to symmetry. The grey areas represent the systematic errors. The data are shown repeatedly and compared to simulated distributions for the reaction chains $\left(J^{P}\right) \rightarrow N(1520) 3 / 2^{-}+\pi^{0}$. Distributions for $\left(1 / 2^{ \pm}\right) \rightarrow N(1520) 3 / 2^{-}+\pi^{0}$ vanish and are not shown. In the fits, the systematic errors are added quadratically to the statistical errors. Picture taken from [80] which provides further details. 
cay fractions into the intermediate orbitally $(\ell=1)$ excited baryon resonances $N(1520) 3 / 2^{-}, N(1535) 1 / 2^{-}$, $N(1680) 5 / 2^{+}$and a pion. At the same time $N(1880) 1 / 2^{+}$, $N(1900) 3 / 2^{+}, N(2000) 5 / 2^{+}$, and $N(1990) 7 / 2^{+}$decay into $N(1520) 3 / 2^{-} \pi, N(1535) 1 / 2^{-} \pi, N(1680) 5 / 2^{+} \pi, N \sigma$ with a significant fraction. Since the individual branching ratios have large error bars [81]: the data were re-fitted using two assumptions:

1. Decays of the four $\Delta^{*}$ 's into $N(1440) \pi, N(1520) \pi$, $N(1535) \pi$, and $N(1680) \pi$ were forbidden. This has little effect on the fit and the $\chi^{2}$ deteriorated by 692 units. We consider this to be at the border of becoming statistically significant. Of course, these decays are not forbidden but obviously, the branching ratios for these decay modes from the four $\Delta^{*}$ 's are small.

2 . If decay modes into orbitally excited states were forbidden for the four $N^{*}$ resonances, the $\chi^{2}$ change became 3880 units and the fit quality deteriorated visibly. The four $N^{*}$ resonances decay via orbitally excited intermediate states with a significant decay fraction.

Why are the decays of the four $N^{*}$ resonances into orbitally excited intermediate resonances so frequent $(\approx 23 \%)$, and why are these decay modes suppressed for the four $\Delta^{*}$ resonances [81]?

These phenomena can be understood inspecting their wave functions. If one neglects mixing, the four positiveparity $\Delta^{*}$-resonances and $N^{*}$-resonances could both be assigned to a spin-quartet with intrinsic orbital and spin angular momenta $L=2$ and $S=3 / 2$. The four positiveparity $\Delta^{*}$ resonances have a spin and a flavor wave function which is symmetric with respect to the exchange of any pair of quarks. The color wave function is completely antisymmetric, hence the spatial wave function $\phi_{n, l}$ must be symmetric as well:

$$
S=\frac{1}{\sqrt{2}}\left\{\left[\phi_{0 s}(\vec{\rho}) \times \phi_{0 d}(\vec{\lambda})\right]+\left[\phi_{0 d}(\vec{\rho}) \times \phi_{0 s}(\vec{\lambda})\right]\right\}^{(L=2)},
$$

with $\rho$ and $\lambda$ being the oscillators which can be excited in the 3-quark-system. For the nucleon states a mixed symmetry spatial wave function is required: For $L=2$ the wave functions have equal admixtures of

$$
\begin{aligned}
\mathcal{M}_{\mathcal{S}} & =\frac{1}{\sqrt{2}}\left\{\left[\phi_{0 s}(\vec{\rho}) \times \phi_{0 d}(\vec{\lambda})\right]-\left[\phi_{0 d}(\vec{\rho}) \times \phi_{0 s}(\vec{\lambda})\right]\right\}^{(L=2)} \\
\mathcal{M}_{\mathcal{A}} & =\left[\phi_{0 p}(\vec{\rho}) \times \phi_{0 p}(\vec{\lambda})\right]^{(L=2)}
\end{aligned}
$$

and both parts need to be present to fulfill the Pauli principle. The part $\mathcal{M}_{\mathcal{A}}$ describes a component in which the $\rho$ and the $\lambda$ oscillator are both excited simultaneously. We now assume that the $\mathcal{M}_{\mathcal{A}}$-part can only de-excite in a twostep process: In the first step one of the intrinsic oscillators is de-excited, and the intermediate state still carries an intrinsic excitation, an intermediate resonance is created. The latter excitation requires a second decay, from the intermediate resonance to the ground state. The large contribution of cascade decays to decays of $N^{*}$ in the fourth resonance region and their smallness in $\Delta^{*}$ decays provide evidence that two independent oscillators are excited in high-mass baryon resonances.
Table 2. Branching ratios BR for $N^{*} \rightarrow N \eta$ decays and the photon helicity amplitudes $A_{1 / 2}, A_{3 / 2}$ for four of the contributing nucleon resonances (taken from [82]). The helicity amplitudes are given in units of $\mathrm{GeV}^{-1 / 2}$. Small numbers below the BRs or below the helicity amplitudes give either the RPP [87] estimate or the entries reported in [49].

\begin{tabular}{|cc|cc|}
\hline \hline Res. & $\mathrm{BR}\left(N^{*} \rightarrow N \eta\right)$ & Res. & $\mathrm{BR}\left(N^{*} \rightarrow N \eta\right)$ \\
$A_{1 / 2}$ & $A_{3 / 2}$ & $A_{1 / 2}$ & $A_{3 / 2}$ \\
\hline$N(1535)$ & $0.42 \pm 0.04$ & $N(1710)$ & $0.25 \pm 0.09$ \\
$1 / 2^{-}$ & $0.42 \pm 0.10$ & $1 / 2^{+}$ & $0.10-0.30$ \\
$0.093 \pm 0.009$ & - & $0.040 \pm 0.020$ & - \\
$0.115 \pm 0.015$ & - & $0.035 \pm 0.012$ & - \\
\hline$N(1650)$ & $0.32 \pm 0.04$ & $N(1720)$ & $0.03 \pm 0.02$ \\
$1 / 2^{-}$ & $0.05-0.15$ & $3 / 2^{+}$ & $0.021 \pm 0.014$ \\
$0.032 \pm 0.006$ & - & $0.115 \pm 0.045$ & $0.135 \pm 0.040$ \\
$0.045 \pm 0.010$ & - & $0.100 \pm 0.020$ & $0.150 \pm 0.030$ \\
\hline \hline \multicolumn{4}{|c}{} \\
\hline \hline
\end{tabular}

\subsection{Negative-parity resonances}

\subsubsection{Negative-parity resonances below $1800 \mathrm{MeV}$}

The five negative-parity nucleon and the two $\Delta$ excitations in the first excitation shell are well established and their properties well defined. However, this does not exclude surprises when new data become available.

New single and double polarization data for the $\gamma p \rightarrow p \eta$ final state were reported recently. The observables $E, G, T, P$, and $H$ have been measured with the CBELSA/TAPS experiment ([82], see project A.1) at ELSA. A few bins of the newly measured double polarization observables $T$ and $E$ are shown in Fig. 7. Data on $T$ and $F$ [83] were obtained in Mainz and data on $E$ have been published recently by CLAS [84]. The latter data are consistent with our CBELSA/TAPS-data but were not yet included in our fits.

The predictions for the newly measured observables $E$ and $T$ from the different PWAs are also shown in Fig. 7. None of the predictions comes close to the data, even though they do describe reasonably well all $\eta$-photoproduction data $(d \sigma / d \Omega, \Sigma)$ which existed before. This clearly shows that $\eta$-photoproduction was less constrained by existing data than $\pi$-photoproduction. Of course, this was to be expected since $\pi N$-elastic scattering does already provide significant information on the $\pi N$ coupling of resonances, while comparable data are missing for the $\eta N$-final state: data on $\pi N \rightarrow \eta N$ are scare.

Table 2 presents the $N^{*} \rightarrow N \eta$ branching ratios of four resonances used in the fit. There are a few interesting observations:

While the $N(1535) 1 / 2^{-} \rightarrow N \eta$ branching ratio is found to be $0.42 \pm 0.04$ (most recent PDG value: $(0.42 \pm$ $\left.0.10) \mathrm{GeV}^{-1 / 2}\right)$, there is a very significant change in the $N(1650) 1 / 2^{-} \rightarrow N \eta$ branching ratio observed. It changes from $0.05-0.15$ (RPP) to $0.32 \pm 0.04$ in our fit. The $N(1710) 1 / 2^{+} \rightarrow N \eta$ branching ratio settles at $0.27 \pm 0.09$, in the higher half of its previous range $0.10-0.30$, while $N(1720) 3 / 2^{+}$contributes little [82]. These results clearly show the power of polarization observables to constrain PWAs; an earlier PWA $[65,85]$ indicated a large $N(1720) 3 / 2^{+}$contributions.

The large $N(1650) 1 / 2^{-} \rightarrow N \eta$ branching ratio found is surprising, given the previously large and puzzling dif- 

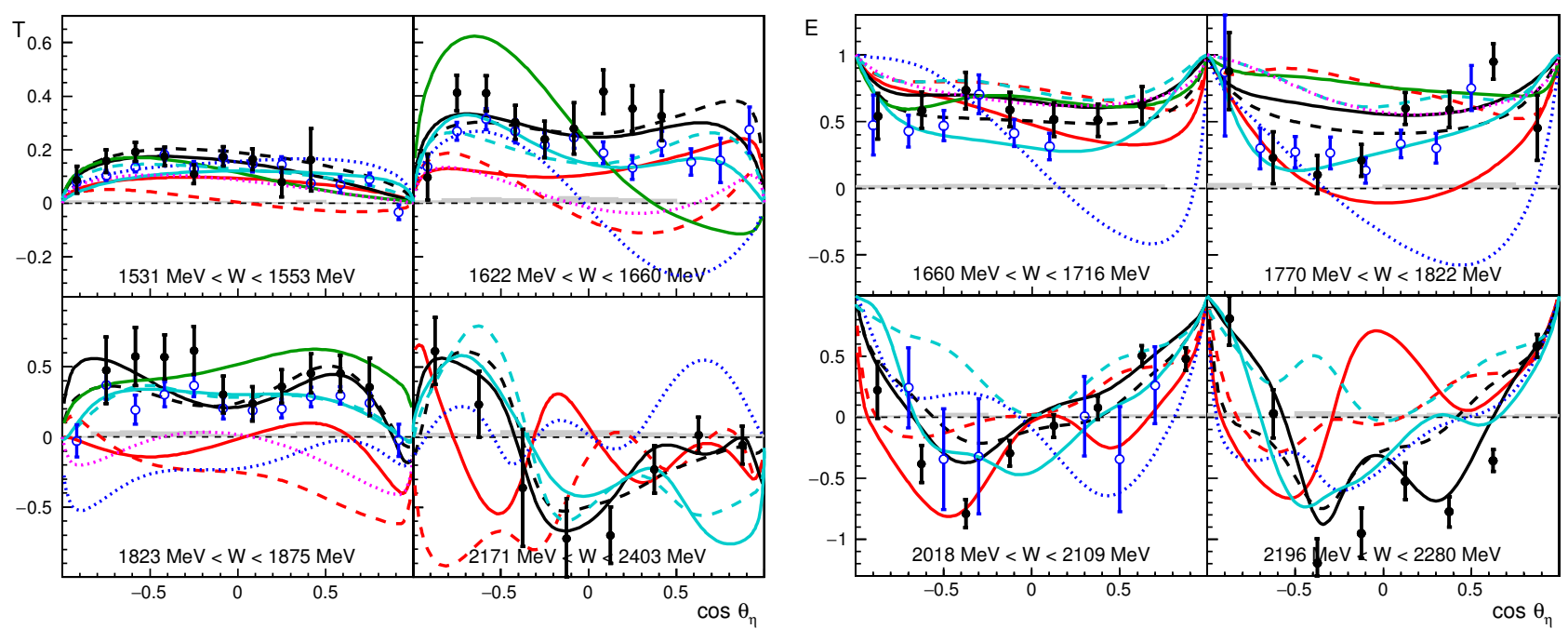

Figure 7. The polarization observable $T$ (left) and $E$ (right) as function of $\cos \theta_{\eta}$ for a few selected W-bins [82] (CBELSA/TAPS-data (black), from [82]). Very recent data from CLAS [84] are shown for comparison as blue open points (due to different binning, the energies differ by up to half of the bin size). The curves represent different models. Black: BnGa fit with (solid) and without (dashed) $N(2200) 5 / 2^{-}$; red solid (dashed): BnGa2011-01 (-02); green: MAID, dark blue (dotted): SAID (GE09); magenta (dotted): Gießen , light blue: JüBo 2015 (dashed) and JüBo 2015-3 [84] (solid).

ference in the $N \eta$ branching ratios of the $N(1535) 1 / 2^{-} /$ $N(1650) 1 / 2^{-}$nucleon-resonance pair which was extensively discussed in literature (see [86]). In the standard quark model, this has been taken as evidence for a large mixing of $\mathrm{SU}(6) \times \mathrm{O}(3)$ states (see Review on Quark Models in the RPP2014 [87]). A further intriguing result is the inversion of the relative sign of the electromagnetic couplings of the $N(1535) 1 / 2^{-}$and the $N(1650) 1 / 2^{-}$state for photoproduction off the proton and the neutron [88].

These results refer to properties of the resonances derived at the pole position. To calculate branching ratios (or Breit-Wigner parameters) from the multi-channel multi-pole K-matrix parameterization we first calculated the couplings of the resonance to the different channels as residues at the pole position. The Breit-Wigner/Flatte couplings were then introduced to be proportional to these couplings with one common factor $f$. The factor $f$ as well as the Breit-Wigner mass are then adjusted until they match exactly the pole position obtained from the full amplitude resulting from the K-matrix-fit to the data. Then, the helicity Breit-Wigner/Flatte couplings were determined making again sure that they reproduce the helicity residues of the full amplitude at the pole position. The resulting branching ratios for four of the contributing resonances are given in Table 2.

It is interesting to note that the $\mathrm{K}$-matrix couplings maintain a small $N(1650) 1 / 2^{-} \rightarrow N \eta$ coupling in the fits and do not show the inversion of the relative sign of $N(1535) 1 / 2^{-}$ and $N(1650) 1 / 2^{-}$electromagnetic couplings.

\subsubsection{Negative-parity resonances above $1800 \mathrm{MeV}$}

We continue the discussion of fits to the double polarization data on $\gamma p \rightarrow \eta p$. The fit did not reproduce well the high-mass range; hence we tried to improve the description by adding one further resonance with spinparity $J^{P}=1 / 2^{ \pm}, \cdots, 9 / 2^{ \pm}$. The best result was achieved
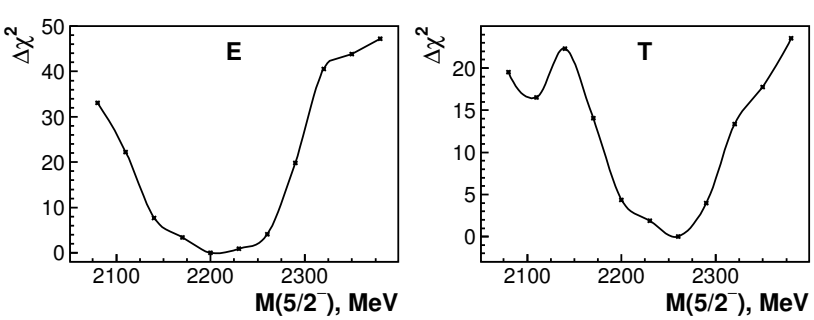

Figure 8. Mass scan for a resonance with $J^{P}=5 / 2^{-}$. Shown is the $\chi^{2}$-change for $E$ and $T$ of the fit as a function of the imposed mass. In the mass scan, starting from the best fit solution, a BreitWigner amplitude is added with its mass fixed while all other parameters are fitted freely. The curves are included to guide the eye. The figure is taken from [82].

by introducing a resonance with $J^{P}=5 / 2^{-}, M=(2200$ $\pm 50) \mathrm{MeV}, \Gamma=(260 \pm 50) \mathrm{MeV}$. Figure 8 shows the $\chi^{2}$ minimum for the two observables $E$ and $T$ if a scan of the $5 / 2^{-}$-resonance mass is performed. In the scan, the mass of the $5 / 2^{-}$-resonance was set to prefixed values while all other parameters were fitted freely. Significant minima are observed. However, no convincing evidence was observed in other reactions. Hence one must consider the

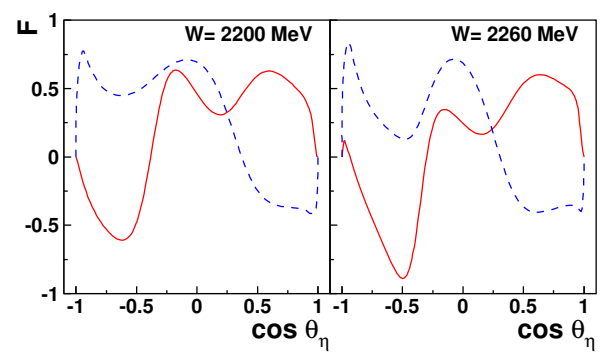

Figure 9. Predictions for the double polarization observable $F$ for $\gamma p \rightarrow p \eta$ : with $N(2200) 5 / 2^{-}$: red solid curve, without $N(2200) 5 / 2^{-}$: blue dashed curve. 
Table 3. Masses and widths of selected negative-parity resonances. The mass values are from [49]. The second column gives the PDG [96] star rating for resonances known in 2010. $\dagger$ : in 2010, this entry summarized observations ranging from 1900 to $2240 \mathrm{MeV}$ and was removed from the RPP in 2012.

\begin{tabular}{lcccc}
\hline \hline$N(1895) 1 / 2^{-}:$ & new & $M_{\mathrm{BW}}=1895 \pm 15$ & $\Gamma_{\mathrm{BW}}=90_{-15}^{+30}$ & {$[\mathrm{MeV}]$} \\
$N(1875) 3 / 2^{-}:$ & new & $M_{\mathrm{BW}}=1880 \pm 20$ & $\Gamma_{\mathrm{BW}}=200 \pm 25$ & {$[\mathrm{MeV}]$} \\
$N(2150) 3 / 2^{-}:$ & new & $M_{\mathrm{BW}}=2150 \pm 60$ & $\Gamma_{\mathrm{BW}}=330 \pm 45$ & {$[\mathrm{MeV}]$} \\
$N(2060) 5 / 2^{-}:$ & new & $M_{\mathrm{BW}}=2060 \pm 15$ & $\Gamma_{\mathrm{BW}}=375 \pm 25$ & {$[\mathrm{MeV}]$} \\
$N(2200) 5 / 2^{-}:$ & $\dagger$ & $M_{\mathrm{BW}}=2200 \pm 50$ & $\Gamma_{\mathrm{BW}}=260 \pm 50$ & {$[\mathrm{MeV}]$} \\
$N(2190) 7 / 2^{-}:$ & $4^{*}$ & $M_{\mathrm{BW}}=2180 \pm 20$ & $\Gamma_{\mathrm{BW}}=335 \pm 40$ & {$[\mathrm{MeV}]$} \\
$N(2250) 9 / 2^{-}:$ & $4^{*}$ & $M_{\mathrm{BW}}=2200 \pm 40$ & $\Gamma_{\mathrm{BW}}=480 \pm 60$ & {$[\mathrm{MeV}]$} \\
\hline \hline
\end{tabular}

evidence for this new resonance with some caution. Based on the PWA-solutions with and without the $N(2200) 5 / 2^{-}$resonance, the double polarization observable $F$ has been predicted. Fig. 9 shows the sensitivity of this observable. Measuring $\mathrm{F}$ would definitely help to settle the question whether or not a $5 / 2^{-}$-resonance exists in a mass range around $2200 \mathrm{MeV}$. Of course also improved statistics on the already measured observables can be expected to lead to an improved sensitivity.

New high-mass negative-parity resonances were reported in [90]. $N(1875) 3 / 2^{-}$proved to be particularly sensitive to the reaction $\gamma p \rightarrow \Sigma^{+} K_{S}^{0}$ [91-93] studied in project B.1. Also, the data on the beam asymmetry on $\pi^{0}$ and $\eta$ have to be mentioned [94, 95]. Evidence for the new resonances can be seen in the mass scans shown in Fig. 10. The masses of high-mass negative-parity resonances as obtained within the BnGa multichannel PWA are collected in Table 3 .
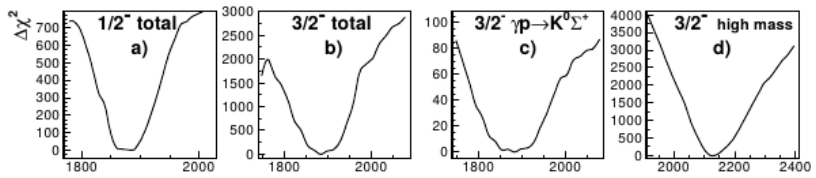

Figure 10. a) Mass scan for a $N_{1 / 2-}$ resonance; change of the total $\chi^{2}$ of the fit as a function of the assumed mass; $\left.b, c\right)$ Mass scan for a $N_{3 / 2^{-}}$resonance; $\chi^{2}$ of the fit as a function of the assumed mass for an assumed width of $100 \mathrm{MeV}$. b) total $\chi^{2}$, c) $\chi^{2}$ contribution from $\gamma p \rightarrow \Sigma^{+} K_{S}^{0}[92,93]$. d) Mass scan for a fourth $N_{3 / 2^{-}}$resonance when $N(1875) 3 / 2^{-}$is included in the K-matrix. The figure is taken from [90].

\subsection{Assignment to SU(6)xO(3)-multiplets}

An assignment of resonances to $\mathrm{SU}(6) \mathrm{xO}(3)$ multiplets is discussed by Klempt and Metsch in [97]. Figure 11 shows the resonances which have at least 2 stars in the RPP and their assignment to $\mathrm{SU}(6) \mathrm{xO}(3)$ multiplets. Resonances where the RPP'2014 states "Latest GWU analysis [15] finds no evidence for the resonance" are indicated by blue / light blue crosses. All states marked by blue, red, or magenta boxes have been identified by the BnGa-PWA.

\subsubsection{Negative parity resonances}

The first excitation shell $(1 \hbar \omega)$ is (since long) complete. The high-mass negative-parity resonances are collected in Table 4. If the three $\Delta^{*}$ resonances $\Delta(1900) 1 / 2^{-}$, $\Delta(1940) 3 / 2^{-}, \Delta(1930) 5 / 2^{-}$are interpreted as a spin triplet, they have a fully symmetric spin and flavor wave function; hence their spatial wave function must be symmetric, too. Thus, they belong to a 56 representation, and there must be a doublet of negative-parity nucleon resonances close in mass. These are identified with $N(1895) 1 / 2^{-}$and $N(1875) 3 / 2^{-}$. These five resonances can be assigned to the third excitation shell with $L_{3 q}=1$. They have one unit of radial excitation $N=N_{\rho}+N_{\lambda}=1$ ).

There are five negative-parity nucleon resonances $N(2150) 3 / 2^{-} ; N(2200) 5 / 2^{-} ; N(2060) 5 / 2^{-} ; N(2190) 7 / 2^{-}$; $N(2250) 9 / 2^{-}$in the mass range from 2050 to $2250 \mathrm{MeV}$. It is tempting to organize these resonances in the same way as those in the first excitation shell, as quartet with an intrinsic quark spin $S=3 / 2$, and a $S=1 / 2$ doublet. In this interpretation, one resonance with $J^{P}=7 / 2^{-}$is missing. We have added to Table $4 \Delta(2223) 5 / 2^{-}$[15], a very wide resonance which did not enter the RPP. It would combine with $\Delta(2200) 7 / 2^{-}$to the expected spin doublet.

\subsubsection{Positive parity resonances}

Well known are the four $\Delta^{*}$-resonances, $\Delta(1910) 1 / 2^{+}$, $\Delta(1920) 3 / 2^{+}, \Delta(1905) 5 / 2^{+}$, and $\Delta(1905) 5 / 2^{+}$, as well as the doublet of $N^{*}$-states, $N(1720) 3 / 2^{+}$and $N(1680) 5 / 2^{+}$ which are except the 3 -star $\Delta(1920) 3 / 2^{+}$all 4 -star resonances. In addition to these states, the $\mathrm{BnGa}$ analysis gives evidence for the existence of four to six positiveparity resonances $N(1880) 1 / 2^{+}, N(1900) 3 / 2^{+}$(with a $N(1960) 3 / 2^{+}$companion, see below), $N(1860) 5 / 2^{+}$(weak evidence from $\mathrm{BnGa}$ but confirmed by other analyses), $N(2000) 5 / 2^{+}$, and $N(1990) 7 / 2^{+}$[49]. $N(1880) 1 / 2^{+}$ was also reported in $[63,98]$. The evidence for the $N(1900) 3 / 2^{+}$has been confirmed by the analyses of other groups, see [63, 98-100], and by the Gießen group [60, 101] within project B.7. In [102], $N(1900) 3 / 2^{+}$was tentatively interpreted as a member of a spin quartet, an identification which is challenged by later results (see below). The other spin partners $N(2000) 5 / 2^{+}$and $N(1990) 7 / 2^{+}$ 


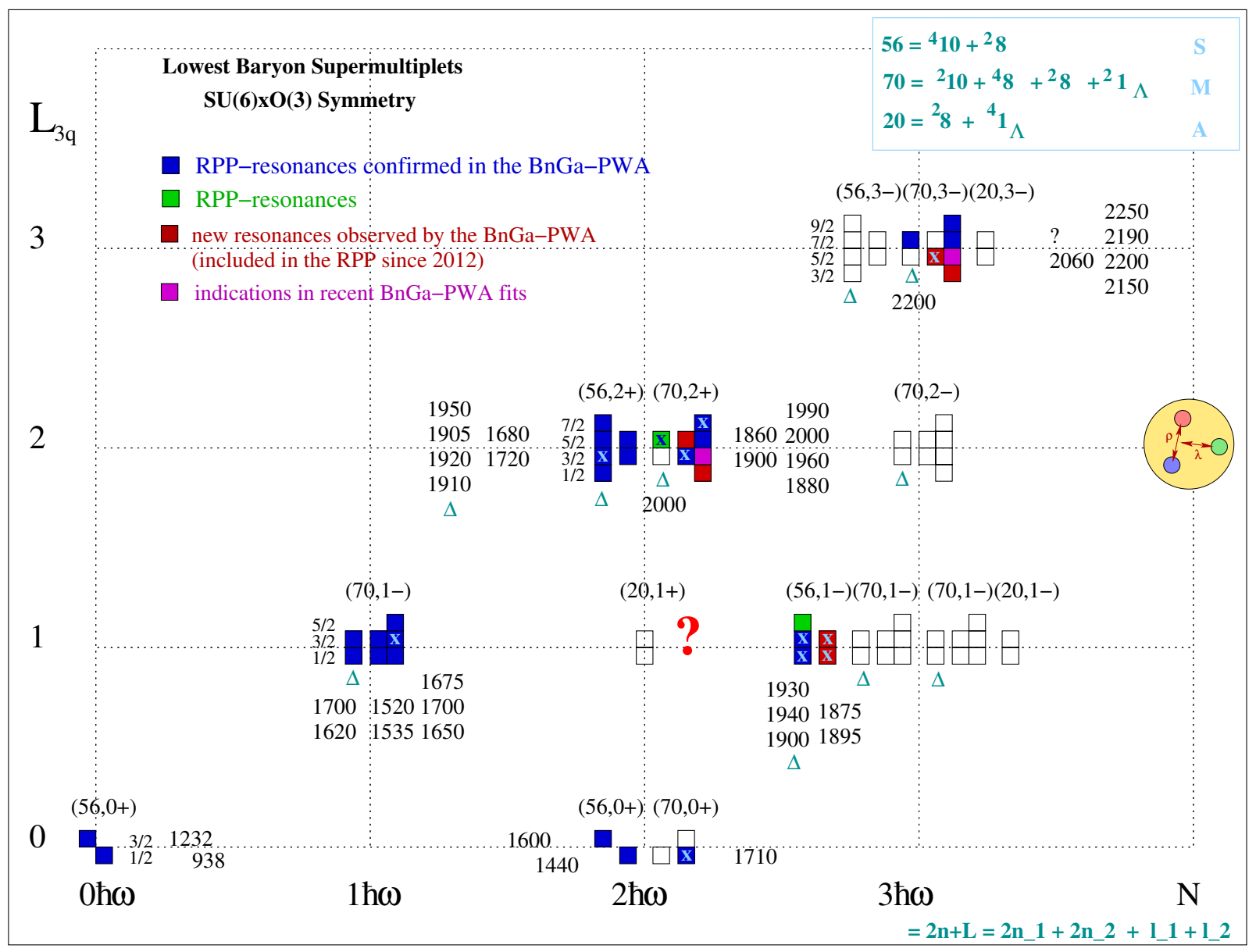

Figure 11. $N^{*}$ - and $\Delta^{*}$-resonances assigned to $\mathrm{SU}(6) \mathrm{xO}(3)$-multiplets. The assignments are not unambiguous and, likely, mixing occurs. Only resonances which have at least a 2-star-assignment in the RPP2014 are shown. The numbers given at the left, right or below the multiplets correspond to the masses of the states. Above the multiplets, the SU(6) multiplet is given together with the total orbital angular momentum $L$ between the quarks and the parity of the multiplet $\left(D, L_{\mathrm{N}}^{P}\right.$, where $D$ is the $\mathrm{SU}(6)$ dimensionality, $L$ is the intrinsic orbital angular momentum, $P$ the parity and $\mathrm{N}$ the shell number of the harmonic oscillator). The light blue or blue crosses indicate the states where the RPP2014 states "Latest GWU analysis [15] finds no evidence for the resonance".

Table 4. Nucleon and $\Delta$ resonances assigned to the third excitation shell. The masses of nucleon resonances are from our work, most $\Delta$ resonances from [96], one from [15]. $N(2190) 7 / 2^{-}$could belong to the $S=3 / 2$ quartet or to a $S=1 / 2$ doublet. $N(2190) 7 / 2^{-}$may both consist of two unresolved resonances, one belonging to the quartet, the other one to the doublet.

\begin{tabular}{llllll}
\hline \hline \multirow{2}{*}{$L=1, N=1$} & $\mathrm{~S}=\frac{1}{2}$ & $N(1895) 1 / 2^{-}$ & $N(1875) 3 / 2^{-}$ & & \\
& $\mathrm{S}=\frac{3}{2}$ & $\Delta(1900) 1 / 2^{-}$ & $\Delta(1940) 3 / 2^{-}$ & $\Delta(1930) 5 / 2^{-}$ & \\
\hline & $\mathrm{S}=\frac{3}{2}$ & $N(2150) 3 / 2^{-}$ & $N(2220) 5 / 2^{-}$ & $N(2190) 7 / 2^{-}$ & $N(2250) 9 / 2^{-}$ \\
$L=3, N=0$ & $\mathrm{~S}=\frac{1}{2}$ & & $N(2060) 5 / 2^{-}$ & & \\
& $\mathrm{S}=\frac{1}{2}$ & & $\Delta(2223) 5 / 2^{-}$ & $\Delta(2200) 7 / 2^{-}$ & \\
\hline \hline
\end{tabular}

were already known since long, even though reported mass values scattered wildly.

In the $3 / 2^{+}$-sector, a much better fit is achieved in the $\mathrm{BnGa}$ analysis when a second $N 3 / 2^{+}$resonance is assumed to exist in addition to the $N(1900) 3 / 2^{+}$-resonance. Its mass is not well defined, the most likely value is $1960 \mathrm{MeV}$. Clearly, quark models have ample possibilities to house two $\mathrm{N} 3 / 2^{+}$resonance at this mass. Expected is, e.g., a 70-plet which contains a quark-spin quartet and a doublet. These six expected states could be the six resonances seen in the BnGa PWA.

The assignment to a quark-spin quartet or a doublet is possible considering the electric couplings. The photocouplings of baryon resonances are mostly given as helicity amplitudes $A_{1 / 2}, A_{3 / 2}$, and for excitations off protons, we gave these quantities only [103]. In a fit to data on photoproduction off neutrons, we evaluated also the electric and magnetic multipole amplitudes [104]. In many cases, 
Table 5. The ratio of the electric and magnetic multipole amplitudes $E / M$ for resonances in the fourth resonance region. For resonances with $J^{P}=1 / 2^{ \pm} E / M$ ratio cannot be determined since they are excited by magnetic quadrupole or electric dipole amplitudes only.

\begin{tabular}{|c|c|c|c|}
\hline & $N(1960) 3 / 2^{+}$ & $N(2000) 5 / 2^{+}$ & $N(1990) 7 / 2^{+}$ \\
\hline$E / M$ & $0.15 \pm 0.12$ & $0.4_{-0.4}^{+0.5}$ & $0.23 \pm 0.17$ \\
\hline & $N(1900) 3 / 2^{+}$ & $N(1860) 5 / 2^{+}$ & \\
\hline$E / M$ & $3.2 \pm 1.9$ & undefined & \\
\hline
\end{tabular}

the ratio is not well defined. Often, the electric multipole amplitude is larger than the magnetic one: $E>M$ holds for many resonances. But there are a few cases where the magnetic multipole prevails over the electric one. A well known example is, of course, $\Delta(1232)$ where a spin flip is required to go from the nucleon to the $\Delta(1232)$ resonance. The $E / M$ ratio is also small for $N(1960) 3 / 2^{+}$, for $N(1990) 7 / 2^{+}$, and, perhaps, for $N(2000) 5 / 2^{+}$. Hence we conjecture that these three resonances belong to a spinquartet while $N(1900) 3 / 2^{+}$belongs to a spin-doublet. For $N(1860) 5 / 2^{+}$, the $E / M$ ratio cannot yet be defined.

These findings are supported by Montagne and Stancu [105] who analyze the nucleon excitation spectrum in an $1 / N_{c}$ expansion scheme. They expect a spin quartet in the $\mathrm{SU}(6)$ multiplet ${ }^{4} N\left[70,2^{+}\right]$with masses

$$
\begin{array}{cccc}
2080 \pm 39 & 2042 \pm 41 & 1955 \pm 32 & 1878 \pm 34 \\
N(1990) 7 / 2^{+} & N(2000) 5 / 2^{+} N(x x x) 3 / 2^{+} & N(1880) 1 / 2^{+} .
\end{array}
$$

The calculated mass values are used to identify candidates which are observed experimentally. These are listed in the second line. For the $3 / 2^{+}$state, Montagne and Stancu predict $1955 \mathrm{MeV}$, and we identify this missing resonance with $N(1960) 3 / 2^{+}$.

Likewise, the $\mathrm{SU}(6)$ multiplet ${ }^{2} N\left[70,2^{+}\right]$is expected with masses

$$
\begin{array}{cc}
1959 \pm 29 & 1902 \pm 22 \\
N(1860) 5 / 2^{+} & N(1900) 3 / 2^{+}
\end{array}
$$

and the identification in [105] with known states is again listed in the second line.

In the second excitation shell, a large fraction of the predicted resonances has been found. Missing are a second $\Delta^{*}$ with spin-parity $J^{P}=3 / 2^{+}$at about 1900 $2000 \mathrm{MeV}$, the doublet partner of $\Delta^{*}(2000) 5 / 2^{+}$(2-starstate, not confirmed by the BnGa-PWA) and the $3 / 2^{+}$companion of $N(1710) 1 / 2^{+}$. Also the one-star $\Delta(1750) 1 / 2^{+}$is not shown, the evidence for its existence is poor only.

Summarizing the discussion of the multiplet structure, we can conclude that the number of resonances is well predicted by the quark model based on $\mathrm{SU}(6) \mathrm{xO}(3)$ symmetry. Of course significant deficiencies are present if quark model predictions of masses or decay widths are compared to the experimental findings. A few entries are missing in the second excitation shell but the resonances found so far provide a reasonable coverage of the expected spectrum. Empty are, however, the entries for the $\left(20,1^{+}\right)$ multiplet. Possible reasons for their absence will be discussed in the Outlook (Section 4).

\subsection{What is the dynamical reason for the existence of parity doublets?}

Quark models use constituent light-quark masses of about $350 \mathrm{MeV}$ to reproduce the masses of ground-state baryons. However, low-energy approximations of QCD [106] lead to the Gell-Mann-Oakes-Renner relation [107] which assigns a mass of a few $\mathrm{MeV}$ to light (current) quarks. The mass gap between current and constituent quarks is interpreted by spontaneous breaking of the chiral symmetry expected for nearly massless quarks $[108,109]$. With no spontaneous symmetry breaking, the masses of positive and negative-parity hadrons would be degenerate in mass when they have the same total angular momentum: the nucleon and its chiral partner $N(1535) 1 / 2^{-}$would have the same mass.

In the higher mass region, parity doublets are observed and often, nucleon and $\Delta$ resonances of given spin and parity form a quartet of almost mass-degenerate states. (One particular case is studied in $[110,111])$. This observation has led to the conjecture that chiral symmetry might be restored effectively in highly excited baryons [38]. The mass generation mechanism in excited hadrons is, according to Glozman [112], very different compared to the mechanism in the lower-mass states. In the latter states, the mass is supposed to be driven by chiral symmetry breaking in the vacuum. For highly excited states, the quark condensate is believed to be almost irrelevant and the mass of resonances within a parity doublet could have a chirally symmetric origin.

This idea is best tested in baryons. In meson spectroscopy, resonances falling onto a leading Regge trajectory (with $J=L+S$ ) have no mass-degenerate parity partner (see Fig. 55 in the review of Klempt and Zaitsev [113]). However, in $\bar{p} p$ formation of, e.g., $f_{4}(2050), L=3$ is required; formation of its (unobserved or non-existing) mass-degenerate parity partner $\eta_{4}$ requires $L=4$. The non-observation of a mass-degenerate $\eta_{4}$ might be due to a suppression by the angular-momentum barrier. In $\pi N$ scattering, $\Delta(1950) 7 / 2^{+}$formation requires $L=3$ and formation of its $7 / 2^{-}$parity partner $L=4$.

This is different in photoproduction of baryons. In photoproduction, a $7 / 2^{+}$resonance needs an electric or magnetic $E_{4}^{+} / M_{4}^{+}$amplitude (with $L=4$ between photon and nucleon); a $7 / 2^{-}$resonance needs a $E_{3}^{-}$or $M_{3}^{-}$ amplitude. Photoproduction hence provides the best and possibly the only chance to find a decisive support or an experimental argument against the hypothesized restoration of chiral symmetry.

Slightly above $1900 \mathrm{MeV}$, four positive-parity and three negative-parity $\Delta^{*}$ resonances have been observed:

\section{$\Delta(1910) 1 / 2^{+} \quad \Delta(1920) 3 / 2^{+} \quad \Delta(1905) 5 / 2^{+} \quad \Delta(1950) 7 / 2^{+}$ $\Delta(1900) 1 / 2^{-} \quad \Delta(1940) 3 / 2^{-} \quad \Delta(1930) 5 / 2^{-} \quad ?$}

New photoproduction data on $T, P, H[115,116]$ $E$ [117], and $G$ [81, 118] for $\gamma p \rightarrow \pi^{0} p$ taken within the A.1 project at Bonn and data on $E$ for $\gamma p \rightarrow \pi^{+} n$ [119] were included in the multi-channel BnGa-PWA and a large number of fits were performed. The fit required contributions from a $\Delta 7 / 2^{+}$and a $\Delta 7 / 2^{-}$resonance. Figure 12 


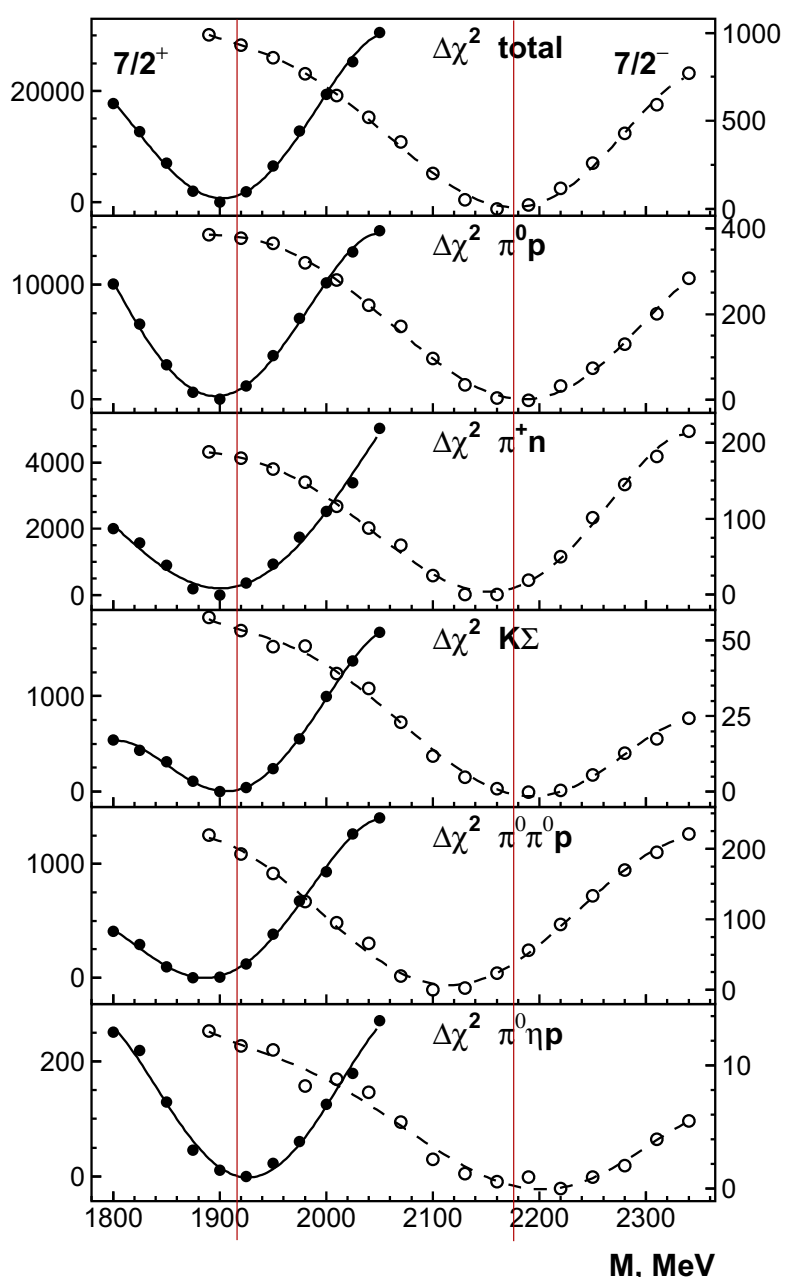

Figure 12. The increase in pseudo- $\chi^{2}$ of the fit to a large body of pion- and photo-produced reactions when the mass of $\Delta(1950) 7 / 2^{+}$(solid points) or $\Delta(2200) 7 / 2^{-}$(open circles) is scanned. The scale on the left (right) abscissa refers to the $7 / 2^{+}$ $\left(7 / 2^{-}\right)$partial wave. The curves are to guide the eye [121]. The red line indicate the mass as obtained by the fit as given in Table 6 .

shows a scan in the $J^{P}=7 / 2^{+}$and $J^{P}=7 / 2^{-}$partial waves. In the mass scans, the $J^{P}=7 / 2^{+}$and $J^{P}=7 / 2^{-}$ partial waves are described by multichannel Breit-Wigner amplitudes rather than by K-matrix amplitudes. Hence the optimal parameters in the scan for mass and width can differ from the optimal values from the main fit. Figure 12 (top) shows the change of the resulting pseudo- $\chi^{2}$ as a function of the imposed mass of the $J^{P}=7 / 2^{+}$or the $J^{P}=7 / 2^{-}$resonance. The total pseudo- $\chi^{2}$ has clear minima at a mass of about $1900 \mathrm{MeV}$ for $J^{P}=7 / 2^{+}$and somewhat below $2200 \mathrm{MeV}$ for $J^{P}=7 / 2^{-}$. The scans demonstrate clearly that the masses of $\Delta(1950) 7 / 2^{+}$and $\Delta(2200) 7 / 2^{-}$are different. Both resonances are seen with compatible masses in $\pi N, K \Sigma, \pi^{0} \pi^{0} p$, and even in $\pi^{0} \eta p$. The properties of the resonances as derived in these fits are listed in Table 6. A $7 / 2^{-}$-state mass degenerate with $\Delta(1950) 7 / 2^{+}$has been found in none of the reactions. The $\Delta(2200) 7 / 2^{-}$observed confirms a 1-star-state given in the
Table 6. Properties of $\Delta(1950) 7 / 2^{+}$and $\Delta(2200) 7 / 2^{-}$: Breit-Wigner masses, widths, helicity amplitudes and branching ratios. The RPP estimates are given as small numbers. The helicity couplings $A_{1 / 2}, A_{3 / 2}$ are given in units of $10^{-3} \mathrm{GeV}^{-1 / 2}$ (see [121]).

\begin{tabular}{|c|c|c|c|}
\hline \multicolumn{2}{|c|}{$\Delta(1950) 7 / 2^{+} \quad * * * *$} & \multicolumn{2}{|c|}{$\Delta(2200) 7 / 2^{-} \quad *$} \\
\hline $\begin{array}{c}M=1917 \pm 4 \\
1915-1950\end{array}$ & $\begin{array}{c}\Gamma=251 \pm 8 \mathrm{MeV} \\
235-335\end{array}$ & $M=2176 \pm 40$ & $\Gamma=210 \pm 70 \mathrm{MeV}$ \\
\hline $\begin{array}{c}A_{1 / 2}=-67 \pm 5 \\
-76 \pm 12\end{array}$ & $\begin{array}{c}A_{3 / 2}=-94 \pm 4 \\
-97 \pm 10\end{array}$ & $A_{1 / 2}=60 \pm 20$ & $A_{3 / 2}=20 \pm 8$ \\
\hline $\begin{array}{c}N \pi 46 \pm 2 \% \\
35-45\end{array}$ & $\underset{\text { seen }}{\Sigma K} 0.6 \pm 0.2 \%$ & $N \pi 3.5 \pm 1.5 \%$ & $\Sigma K \quad 4 \pm 3 \%$ \\
\hline $\begin{array}{c}\Delta \pi \quad 5 \pm 3 \% \\
20-30\end{array}$ & $\Delta \pi \quad \begin{array}{l}- \\
\text { seen }\end{array}$ & $\Delta \pi 70 \pm 15 \%$ & $\Delta \pi 15 \pm 10 \%$ \\
\hline$\Delta \eta 0.3 \pm 0.3 \%$ & $\Delta \eta$ & $\Delta \eta \sim 1 \%$ & $\Delta \eta$ \\
\hline
\end{tabular}
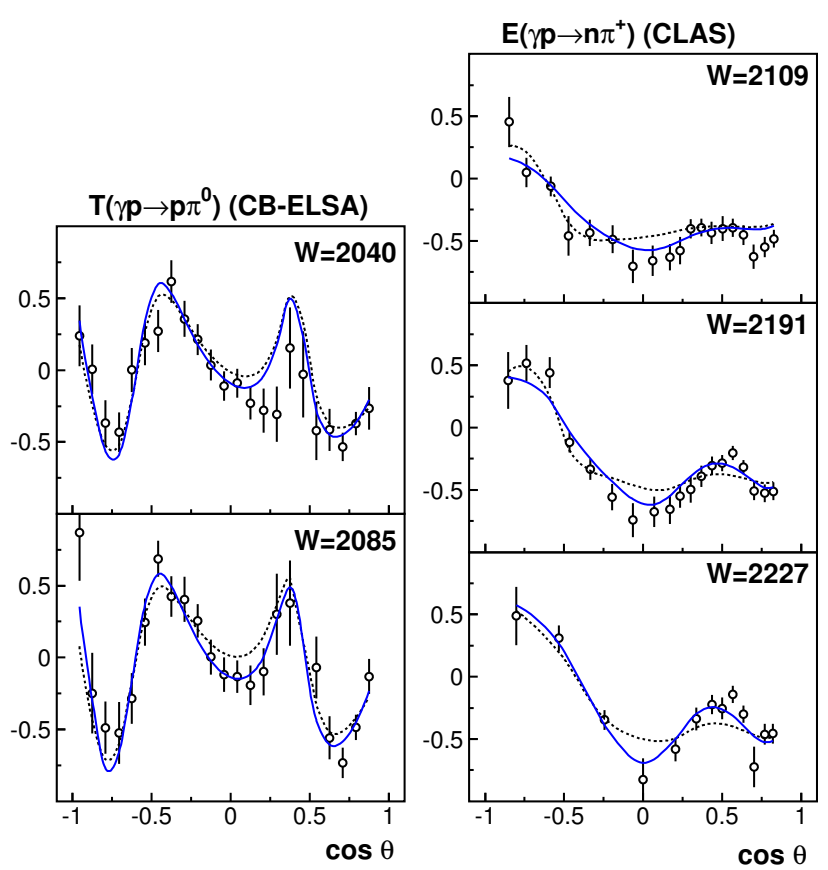

Figure 13. Selected data and fits. Data: Target asymmetry T [115, 116]; helicity dependence $E$ [119]. Best fit: solid curve, fit without $\Delta(2200) 7 / 2^{-}$: dotted curve. The fit deteriorated by $\Delta \chi^{2}=597$ when $\Delta(2200) 7 / 2^{-}$was removed.

RPP. The PDG decided to upgrade $\Delta(2200) 7 / 2^{-}$to a $3^{*}$ resonance [120].

The resonances with $J^{P}=1 / 2^{ \pm}, 3 / 2^{ \pm}, 5 / 2^{ \pm}$are nearly mass-degenerate. The ideas of Glozman require necessarily a $\Delta 7 / 2^{-}$resonance at about the same mass [114]. For such a resonance, there is no evidence at all!

Fig. 13 shows examples for data bins in comparison to the best fit achieved with or without including a negative parity $J=7 / 2, \Delta(2200)$-state. The data description clearly improves if the $7 / 2^{-}$-state is included in the fit. Further examples for polarization observables and more analysis details can be found in [121].

What is then the reason for the often observed parity doublets? Klempt argues that the dynamical reason for the parity doublets lies in the string nature of the quark-quark 


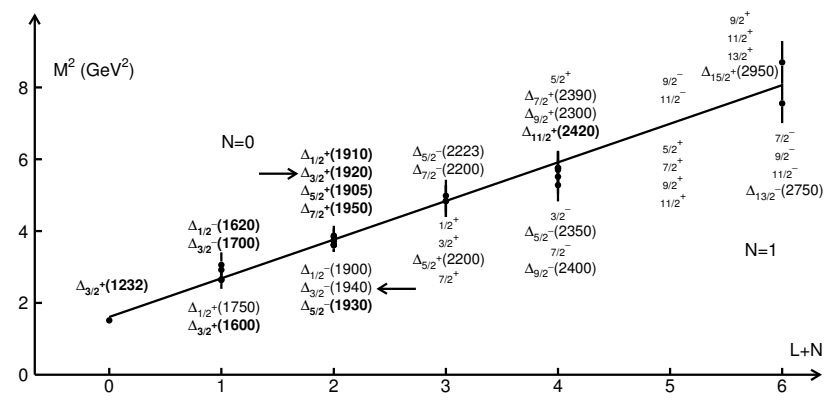

Figure 14. Mass of $\Delta$ resonances as a function of the leading intrinsic orbital angular momentum $L$ and the radial excitation quantum number $N$ (corresponding to $n_{\rho}+n_{\lambda}$ in quark models). Resonances assigned to $N=0$ and $N=1$ are listed above or below the trajectory. The solid line represent a linear trajectory. Figure taken from [126]

interaction. His empirical mass formula [122] was derived in AdS/QCD [123], and was shown to be, compared to quark models requiring a much larger number of parameters, at least twice as precise in calculating baryon masses, using just two parameters [124]. In Ref. [125], Klempt pointed out that the $\Delta 7 / 2^{-}$mass is decisive for the interpretation. In string models, the mass increases with $L+N$ instead of the harmonic oscillator which suggests $L+2 N$ $\left(\vec{L}=\vec{l}_{\rho}+l_{\lambda}, N=n_{\rho}+n_{\lambda}\right)$. Evidence for the string like behavior was presented in [126] from which we take Fig. 14 to demonstrate the $L+N$ dependence of baryon masses. The future will tell us whether the next-generation lattice calculations performed at lower pion masses will provide results closer to the experimental findings: will there be parity doublets for most resonances, except for those on the leading Regge trajectories?

\subsection{Photoproduction of $\omega$ mesons}

At high energies, real or virtual photons interact with protons by diffraction [127]. The photon converts into a vector meson $\left(\rho^{0}, \phi, \omega\right)$ and scatters off the proton by the exchange of Pomerons carrying the quantum numbers of the vacuum $[128,129]$. Close to the $\omega$ production threshold, $N^{*}$ resonances are likely to contribute to the reaction [130, 131]. The Gießen group pioneered the PWAs of the reactions $\pi^{-} p \rightarrow \omega n$ and $\gamma p \rightarrow \omega p$ [60], see project B.7.

The CLAS collaboration reported a high-statistics study of $\omega$ photoproduction $[133,134]$ and claimed evidence for several $N^{*}$ s decaying into $N \omega$. The A2collaboration at MAMI reported differential cross sections from threshold to $E_{\gamma}=1.4 \mathrm{GeV}$ with a $15 \mathrm{MeV}$ binning and full angular coverage [135]. No resonant contributions were discussed. The CBELSA/TAPS collaboration measured $d \sigma / d \Omega$ and spin density matrix elements [136], the beam asymmetry $\Sigma$ [132], the helicity difference $E$, and the correlation $G$ between linear photon and longitudinal target polarization [137]. Photoproduction of $\omega$ mesons off neutrons was studied in [138].

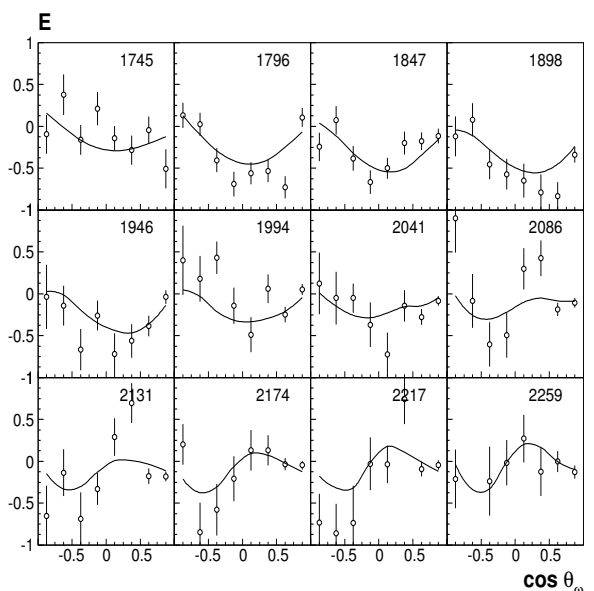

Figure 15. The helicity asymmetry $E$ in bins of the photon energy, taken from [137].

Table 7. Branching ratios (B.R. in \%) for $N^{*} \rightarrow N \omega$ decays from the BnGa PWA, see [62]. Small numbers were reported in [60]. The $\delta\left(\chi^{2}\right)$ values give the change in $\chi^{2}$ when the $N \omega$ decay mode is excluded.

\begin{tabular}{lcclcc}
\hline \hline Resonance & B.R. & $\delta\left(\chi^{2}\right)$ & Resonance & B.R. & $\delta\left(\chi^{2}\right)$ \\
\hline$N(1700) 3 / 2^{-}$ & $22 \pm 12$ & 100 & $N(1900) 3 / 2^{+}$ & $\begin{array}{c}15 \pm 8 \\
13 \pm 9\end{array}$ & 70 \\
$N(1710) 1 / 2^{+}$ & $2 \pm 2$ & 26 & $N(2000) 5 / 2^{+}$ & $\begin{array}{c}18 \pm 8 \\
1 \pm 1\end{array}$ & 42 \\
& $8 \pm 5$ & & & \\
$N(1720) 3 / 2^{+}$ & $26 \pm 14$ & 105 & $N(2060) 5 / 2^{-}$ & $4 \pm 3$ & 37 \\
$N(1875) 3 / 2^{-}$ & $13 \pm 7$ & 98 & $N(2100) 1 / 2^{+}$ & $15 \pm 10$ & 78 \\
& $20 \pm 4$ & & & & \\
$N(1880) 1 / 2^{+}$ & $20 \pm 8$ & 33 & $N(2150) 3 / 2^{-}$ & $12 \pm 8$ & 99 \\
$N(1895) 1 / 2^{-}$ & $28 \pm 12$ & 100 & $N(2190) 7 / 2^{-}$ & $14 \pm 6$ & 131 \\
\hline \hline
\end{tabular}

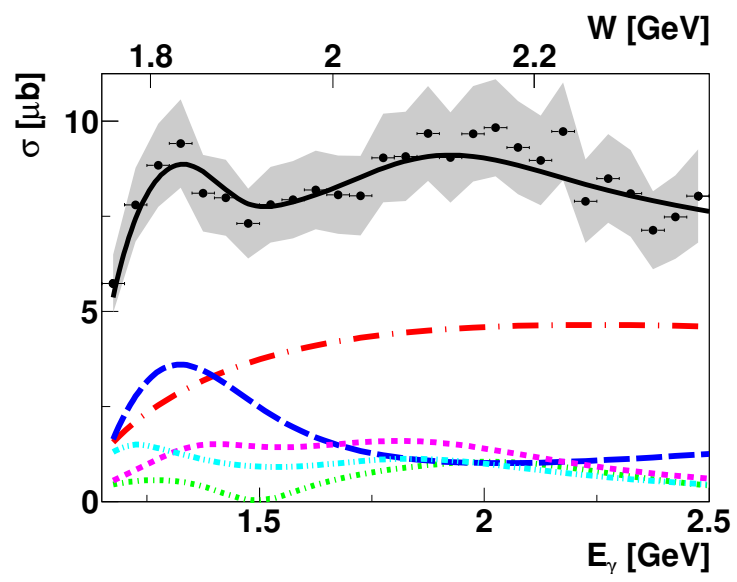

Figure 16. Total cross section for $\gamma p \rightarrow p \omega$ from the CBELSA/TAPS experiment $(\bullet)$ as a function of the initial photon $\left(E_{\gamma}\right)$ and center-of-mass (W) energy, see $[62,136]$. The error bars represent the statistical, the grey band the systematic uncertainty. The Bonn-Gatchina fit is represented by a solid black line. The largest contributions are Pomeron-exchange (red), resonant production of the $J^{p}=3 / 2^{+}$partial wave (blue), $3 / 2^{-}$partial wave (purple), $5 / 2^{+}$partial wave (blue), and $1 / 2^{-}$partial wave (green). 

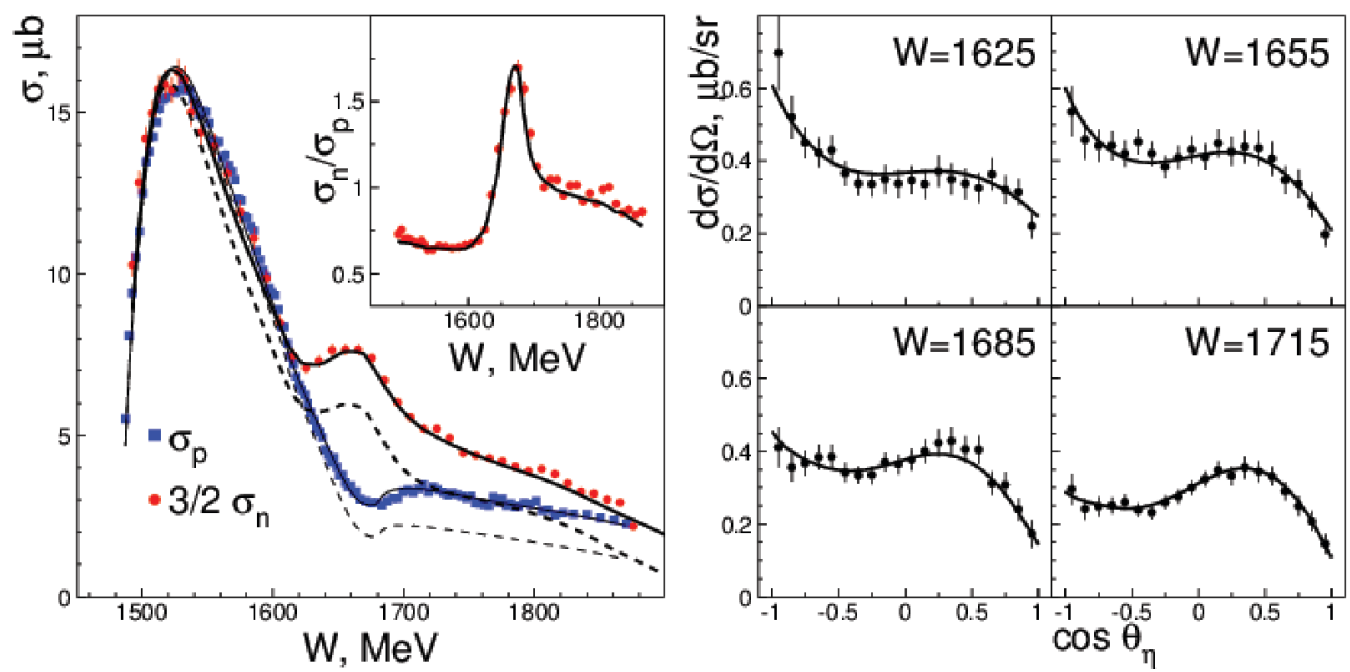

Figure 17. Left: The total cross section for $\gamma n \rightarrow \eta n$ (multiplied by 3/2), $\gamma p \rightarrow \eta p$, and their ratio (as inset) [150] . The solid curves represent our fit [88] folded with the experimental resolution (thick $\eta n$, thin $\eta p$ ), the dashed curves the contributions from the $S_{11}$ waves. Right: Selected differential cross section bins for $\gamma n \rightarrow \eta n$ in the region of the narrow structure.

The data were included in the BnGa data base. Figure 15 shows the helicity difference $E$ with the BnGa fit. The PWA identified twelve $N^{*} \rightarrow N \omega$ branching ratios (see Table 7); most of these were determined for the first time. The total cross section, shown in Fig. 16, is obtained by integration of the differential cross sections. Fig. 16 also shows the dominant partial wave contributions.

Only $N^{*}$ contribute to $\gamma p \rightarrow \omega p . \Delta^{*}$ decays can be studied in the process $\gamma p \rightarrow \pi^{0} \omega p$ to which the isobar $\Delta(1232) \omega$ makes the most significant contribution [139]. Similarly, the reaction $\gamma p \rightarrow K^{*} \Lambda$ has no $\Delta^{*}$ contributions [140]. Instead, the $\mathrm{BnGa}$ PWA finds contributions from $N(1895) 1 / 2^{-}, N(1880) 1 / 2^{+}, N(2100) 1 / 2^{+}$, and two or three higher-mass $N^{*}$ 's with ill-defined quantum numbers. $N^{*}$ and $\Delta^{*}$ both contribute to $\gamma p \rightarrow K^{*} \Sigma$ [141] but a PWA has not been attempted.

\subsection{Is the narrow peak seen in the reaction $\gamma n \rightarrow \eta n$ a resonance?}

The "discovery" in 2003 of $\Theta^{+}(1540)$, an exotic baryon with positive strangeness stimulated further studies [142145] and the search for its non-strange partner. Indeed, a narrow structure was observed in the reaction $\gamma n \rightarrow$ $\eta n$ studied at GRAAL [146]. Its properties, mass of $1685 \mathrm{MeV}$, its narrow width, the production off neutrons but not off protons, and its preference to decay into $\eta n$ would be consistent with predictions for the nonstrange member of a multiplet of pentaquarks with spin-parity $J^{P}=1 / 2^{+}$[142]. The observation attracted considerable interest, and the structure was listed in the RPP as one-star resonance $N(1685)$ [71].

The evidence for the existence of $\Theta^{+}(1540)$ faded away in a number of precision experiments (even though further evidence is reported in several more recent experiments, see [147] for a recent review. In contrast to $\Theta^{+}(1540)$, the peak at about $1685 \mathrm{MeV}$ became increas- ingly significant with better experiments at ELSA and MAMI [148-150], its existence is beyond doubt. But the interpretation as a resonance is unclear.

The Gießen group explained the narrow peak in the $\eta$ photoproduction on the neutron in terms of coupled-channel effects due to $N(1650) 1 / 2^{-}$and $N(1710) 1 / 2^{+}$[151]; Shyam and Scholten interprete the peak by interference effects between the $N(1650) 1 / 2^{-}$, $N(1710) 1 / 2^{+}$, and $N(1720) 3 / 2^{+}$resonances [152] within a coupled-hannel analysis; Döring and Nakayama ascribe the peak to effects from strangeness threshold openings [153]. The Bonn-Gatchina group [154, 155] and Zhong and Zhao [156] demonstrated that the narrow peak can be explained naturally by interference effects in the $J^{P}=$ $1 / 2^{-}$wave, even though - according to $[154,155]$ - solutions with a narrow resonance were not yet excluded by the data.

Fig. 17 shows the total cross section for $\gamma p \rightarrow \eta p$, for $\gamma n \rightarrow \eta n$ and - as insert - the ratio $\sigma_{n} / \sigma_{p}$ from the most recent experiment [150]. The new data exceed the earlier data both in quality and in statistics. In the new experiment, the hit proton or neutron was detected, and hence the $N \eta$ invariant mass was reconstructed without smearing due to the Fermi motion. These experimental achievements greatly enhanced the visibility of the narrow structure. The contributions from $\eta$ photoproduction off neutrons were determined by two different methods; the differences were used to estimate the systematic error.

In [150], the narrow structure was tentatively interpreted as a narrow nucleon resonance at $W=(1670 \pm$ 5) $\mathrm{MeV}$ mass and $\Gamma=(30 \pm 5) \mathrm{MeV}$ width. The product coupling of the hypothesized resonance given by its helicity amplitude $A_{1 / 2}^{n}$ and the branching ratio for its neutron- $\eta$ decay $b_{\eta}$ was determined to $\sqrt{b_{\eta}} A_{1 / 2}^{n}=(12.3 \pm$ 0.8) $10^{-3} \mathrm{GeV}^{-1 / 2}$. 
The data were fitted within the Bonn-Gatchina partialwave analysis [88]. Masses, widths, decay couplings of resonances were all frozen by a fit to $\pi N$ elastic and inelastic reactions and to data on photoproduction off protons (see [49, 159] for the data included). Here, only the helicity amplitudes for the photoproduction of nucleon resonances off neutrons and contributions from $t$ - and $u$ channel exchange were used as free parameters. To constrain the data on $\gamma n \rightarrow \eta n$ further, we included GRAAL data on the beam asymmetry for this reaction [157] and data on $\gamma n \rightarrow \pi N$ [158]. These additional data were described well and have no impact on the conclusions; hence we retain from a more detailed discussion of those data.

The new and precise data are well described by the interference within the $S_{11}$ wave and are now definitely incompatible with the existence of a nucleon resonance with the reported properties. If a narrow resonance with the properties reported in [150] is imposed in the fit, the $\chi^{2}$ of the fit deteriorates very significantly. The reason for the peak structure in $\eta n$ and dip structure in $\eta p$ lies in the opposite relative sign of the helicity amplitudes for the two resonances $N(1535) S_{11}$ and $N(1650) S_{11}$. The narrow structure observed in photoproduction of $\eta$ mesons off neutrons does not support the existence of a nucleon resonance with exotic properties. We mention that data on $\eta^{\prime}$ production off neutrons [89] do not show a similar structure.

\subsection{What is the relation between quark-model resonances and dynamically generated resonances?}

A significant number of nucleon resonances can be generated dynamically from the interaction of their decay products. In coupled-channel dynamical approaches, some baryon resonances need to be put in "by hand", others are generated by the unitarization of the background amplitude. Some of the dynamically generated poles can be identified with quark-model states. But there are poles which do not correspond to quark mode states, and there are resonances which are not (yet?) dynamically generated. The relation between quark-model resonances and dynamically generated resonances remains an unsolved problem in hadron spectroscopy.

\subsection{Photoproduction multipoles from different PWA groups}

Amplitudes are more sensitive when different models are to be compared. In [160] we have reported transition amplitudes to $\Lambda K, \Sigma K$, and $N \eta$ for pion and photo-produced reactions. Fig. 18 shows, as example, the $\mathrm{BnGa}-E_{0^{+}}$ and the $E_{2^{-}}$multipoles for $\pi^{0}$ photoproduction before (2011, red) and after (2014, blue, for further multipoles see $[115,116])$ the inclusion of the new double polarization data on $E, G, T, P$, and $H$.

The error bands for the $\mathrm{BnGa} 2011$ solutions (red) were derived from the $(1 \sigma)$ spread of 12 different solutions with different assumptions on the ingredients: the number of poles in the $J^{P}=3 / 2^{+}$wave was 3 or 4 , in the $J^{P}=5 / 2^{+}$ wave 2 or 3 , the $N(1700) 3 / 2^{-}$width of the pole converged to a wide $(\sim 600 \mathrm{MeV})$ or a narrow $(\sim 250 \mathrm{MeV})$ value, a Kmatrix formalism was used or, alternatively, a $N / D$-based parametrization [62]. The $\chi^{2}$ ranged from its minimum $\chi_{\min }^{2}$ to $\chi_{\text {min }}^{2}+800$. (Note that the absolute $\chi^{2}$ value is meaningless since part of the data are multiparticle final states and fitted in an event-based likelihood fit. The log likelihood value is then converted into a pseudo- $\chi^{2}$ [49].)

In the new fits including the new polarization data, the fits started from the same solutions, then all parameters were re-optimized. All fits converged, but 6 fits resulted in a $\chi^{2}$ larger than the new $\chi^{2}$-minimum $\left(\chi_{\text {new min }}^{2}\right)$ by 1000 units or more. These fits, mostly those with only 3 poles in the $I=1 / 2, J^{P}=3 / 2^{+}$wave, were then removed from the error analysis. The resulting error bands for all remaining solutions within the same $\chi^{2}$-boundaries as for the old solutions are also shown in Fig. 18.

The new error bands (blue) are significantly smaller than the previous ones. Averaged over all multipoles and energies, the errors are reduced by a factor of 2.25 .

Fig. 19 shows the new double-polarization data from JLab and Bonn with fits from MAID, SAID, JüBo, and $\mathrm{BnGa}$, for fits which were made before the data were available (left) and with the new data included (right). Large deviations are observed even at lower photon energies, where one might have expected that $\pi^{0}$-photoproduction is well understood. After a refit, all PWAs are capable to describe the new data.

In Fig. 20 (left) the $E_{0^{+}}$-multipoles from MAID, SAID, JüBo, and BnGa are shown (for further multipoles see [161]). It is striking that even at low energies, large differences between all four solutions are seen. Obviously, the data that existed prior to the double polarization mea-
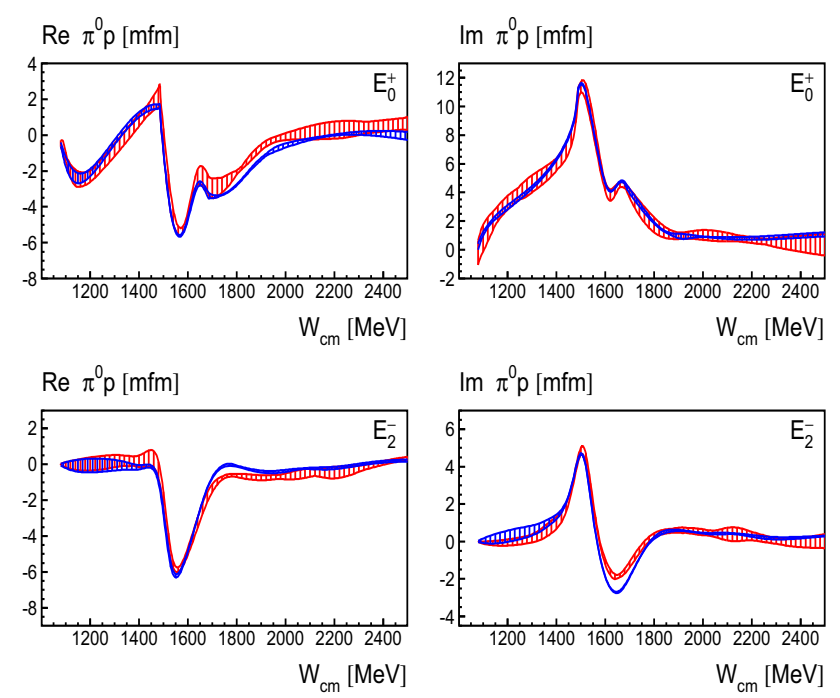

Figure 18. Multipole decomposition of the $\gamma p \rightarrow p \pi^{0}$ transition amplitudes, real part (left), imaginary part (right), showing two examples the $E_{0}^{+}$and the $E_{2}^{-}$-multipole $[115,116]$. The red shaded areas give the range from a variety of different fits derived from solution BnGa2011-01 and BnGa2011-02 [49]. The blue shaded area represents the range of solutions when the new data are included in the fit (BnGa2014). 
surements (e.g. the $p \pi^{0}$-double polarization data discussed in project A.1) did not constrain the solution to a sufficient accuracy. For a further comparison of PWA-predictions to the new polarization data see also the contribution of project A.1 to these proceedings.
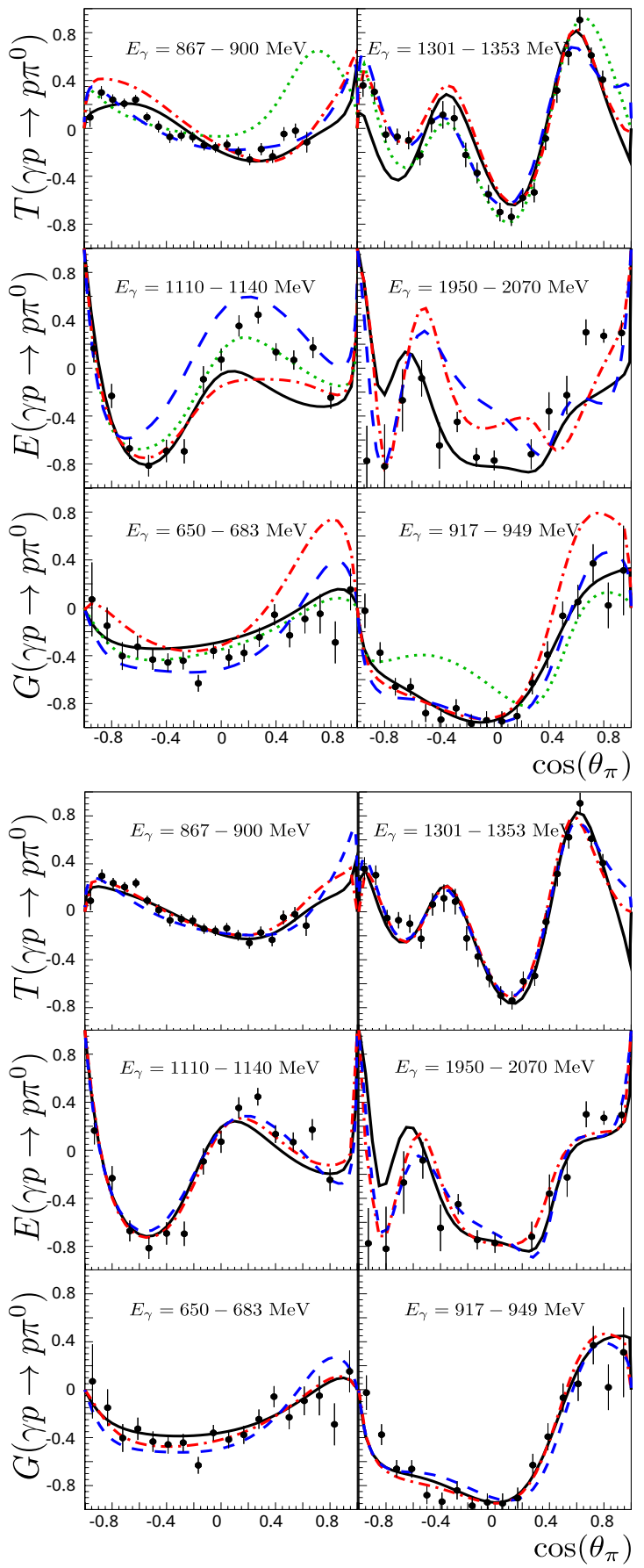

Figure 19. Selected new data, predictions (top), and new fits (bottom) from different PWAs: BnGa2011-02 (black), JüBo2015B (blue), MAID2007 (green), SAID CM12 (red). The predictions are based on fits which did not yet use these new data. Ref. [161] gives a survey of the data included in the different PWAs.

The obvious next step is to include the new data into the other PWAs as well. Since the new data provide additional constraints, a better consistency of the multipoles can be expected. The improvement in the data descrip-
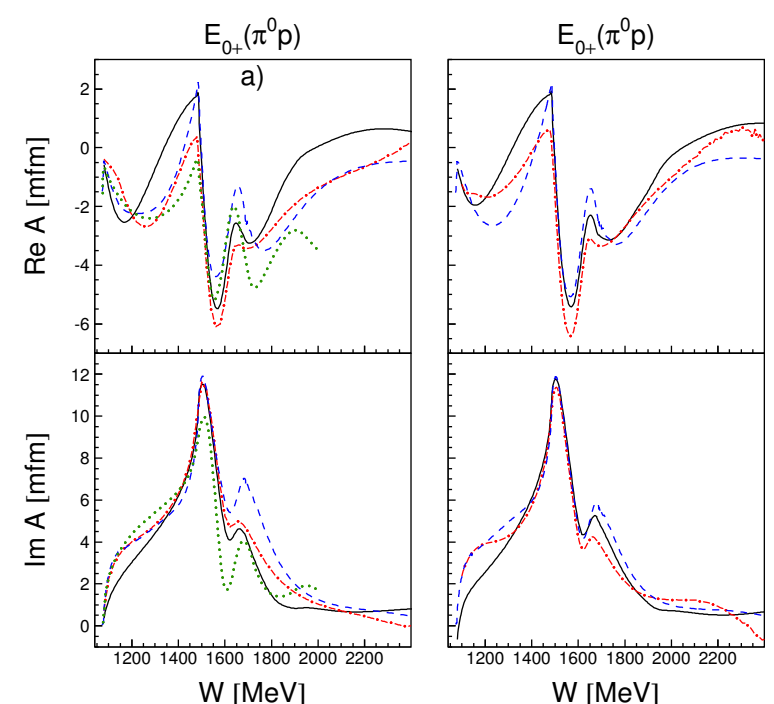

Figure 20. Real (top) and imaginary (bottom) part of the $E_{0}^{+}$-multipole before (left) and after (right) including new data, MAID (green), SAID (red), JüBo (blue), and BnGa (black).

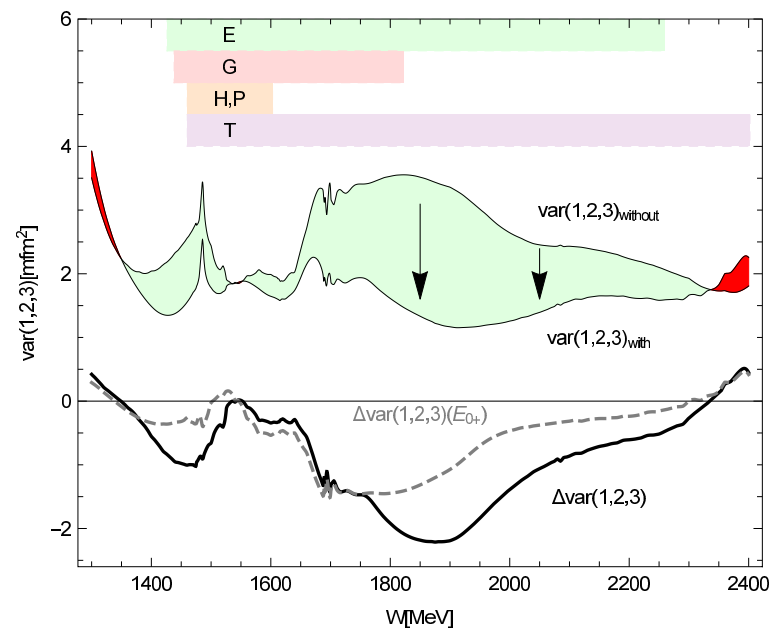

Figure 21. The variance of the BnGa, SAID and JüBo-analyses summed over all $\gamma p \rightarrow p \pi^{0}$ multipoles up to $L=4$. The range covered by the new double polarization observables are indicated by shaded areas. Over the largest part of the energy range the new data have enforced an improvement of the overall consistency. The improvement is displayed as light green area and, separately as difference of the variance. The contribution to the improvement from the $E_{0}^{+}$wave is shown as the dashed curve. Ranges with an overall deterioration are marked in red. For details see [161]

tion is shown in Fig. 19 (bottom). The improvement in consistency of the multipoles is shown in Fig. 20 using the $E_{0}^{+}$-multipole as example. For a complete picture of the multipoles see [161]. Fig. 21 shows the improvement in the overall consistency of the BnGa, SAID and JüBoanalyses considering all $\gamma p \rightarrow p \pi^{0}$ multipoles up to $\mathrm{L}=$ 4. It becomes clearly visible that the new data enforces the multipoles to approach each other, the variance is reduced by about a factor of two. 

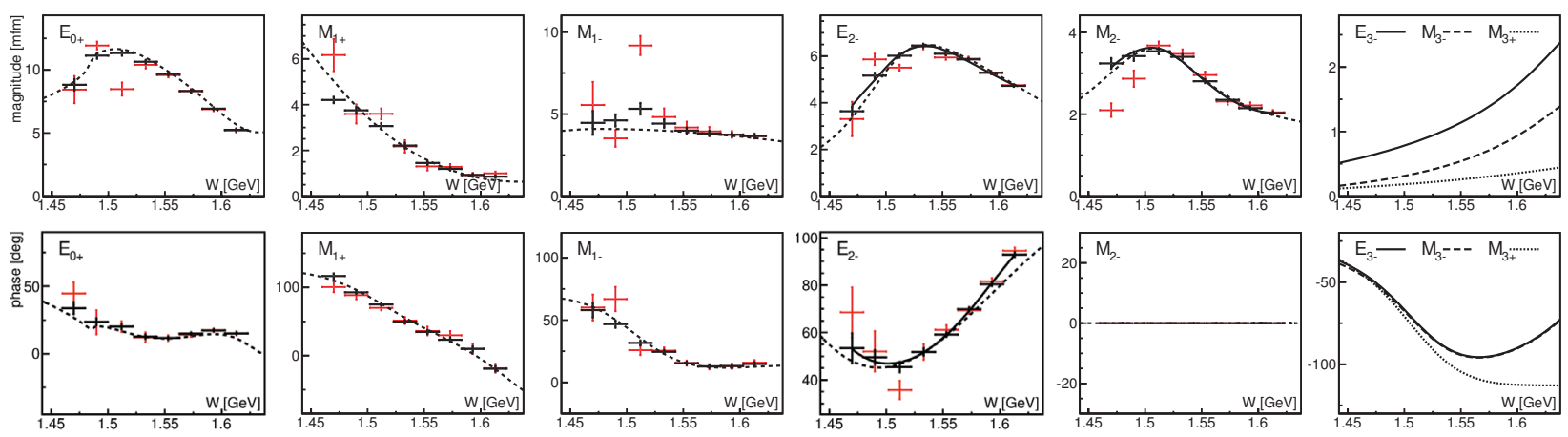

Figure 22. Magnitude (upper row) and phase (lower row) of multipoles derived from a fit to data in slices of energy. Grey (red) crosses show the results of an unbiased fit, black crosses represent a fit with a penalty function (see text). The dashed lines show the new energy-dependent Bonn-Gatchina fit (BnGa2014), the solid lines represent a Breit-Wigner plus background fit to the black crosses for $E_{2^{-}}$and $M_{2^{-}}$. The largest contributions from higher waves (from BnGa2014) are shown as well. The $E_{3^{-}}$and $M_{3^{-}}$multipoles excite the close-by resonance $N(1680) 5 / 2^{+}, M_{3^{-}}$excites $\Delta(1950) 7 / 2^{+}$. Figure taken from [115].

Table 8. The $N(1520) 3 / 2^{-}$helicity amplitudes (in $\mathrm{GeV}^{-1 / 2}$ ) as determined in [115].

\begin{tabular}{lccccc}
\hline$N(1520) 3 / 2^{-}$ & BnGa [115] & CM12 [167] & SN11 [168] & BnGa [49] & PDG [96] \\
\hline$A_{1 / 2}$ & -0.022 & -0.019 & -0.016 & -0.022 & -0.024 \\
& \pm 0.009 & \pm 0.002 & \pm 0.002 & \pm 0.004 & \pm 0.009 \\
$A_{3 / 2}$ & 0.118 & 0.141 & 0.156 & 0.131 & 0.166 \\
& \pm 0.020 & \pm 0.002 & \pm 0.002 & \pm 0.010 & \pm 0.005 \\
\hline
\end{tabular}

\subsection{Can photoproduction multipoles be determined in slices of fixed masses?}

For a long time, the ultimate aim of PWAs of photoinduced reactions was to construct the four CGLN amplitudes from data binned in photon energy (or invariant $\gamma N$ mass) and the meson production angle $\theta$ [162]. It was shown that eight well chosen experiments are required (and sufficient) to determine these amplitudes [163-165], at least when these observables are measured with sufficiently (i.e. extremely) high precision. Ideally, one would expand the constructed amplitude at each mass value in $\cos \theta$-dependent multipoles; the results are then grouped according to their multipole structure and then given as functions of invariant mass. These energy-independent multipoles can than be fitted with dynamical reaction models to separate backgrounds and poles in a given multipole, and to determine the pole properties. However, even with extremely precise data, there is one overall phase per every bin in mass and meson production angle which is difficult to deal with. (One could, e.g., take one partial wave from an energy-dependent fit and determine the other phases relative to this wave.) A more fruitful path is a truncated partial wave analysis [166].

The measurement of single and double-polarization observables for the reaction $\gamma p \rightarrow p \pi^{0}$ were used to reconstruct multipoles with $L=0,1$ and 2 [115]. Multipoles with $L \geq 3$ were fixed to those from the $\mathrm{BnGa}$ energy-dependent partial wave analysis while magnitudes and phases for $E_{0^{+}}, E_{1^{+}}, E_{2^{+}}, E_{2^{-}}, M_{1^{+}}, M_{2^{+}}$, and $M_{1^{-}}$ were left free (see Fig. 22). The helicity amplitudes of $N(1520) 3 / 2^{-}$were thus deduced with minimal model assumptions. The results are collected in Table 8 . They are partly inconsistent with older (model-dependent) determinations and support those of the energy-dependent BnGa PWA.

Production of $\Lambda$ hyperons is particularly fruitful to construct multipoles from the data since their decay reveals their degree of polarization. In a first step, we analyzed the reaction $\pi^{-} p \rightarrow \Lambda K^{0}$ [169] and determined low-lying multipoles. Ambiguities and large fluctuations showed up. Consistent results for the $S_{11}, P_{11}$, and
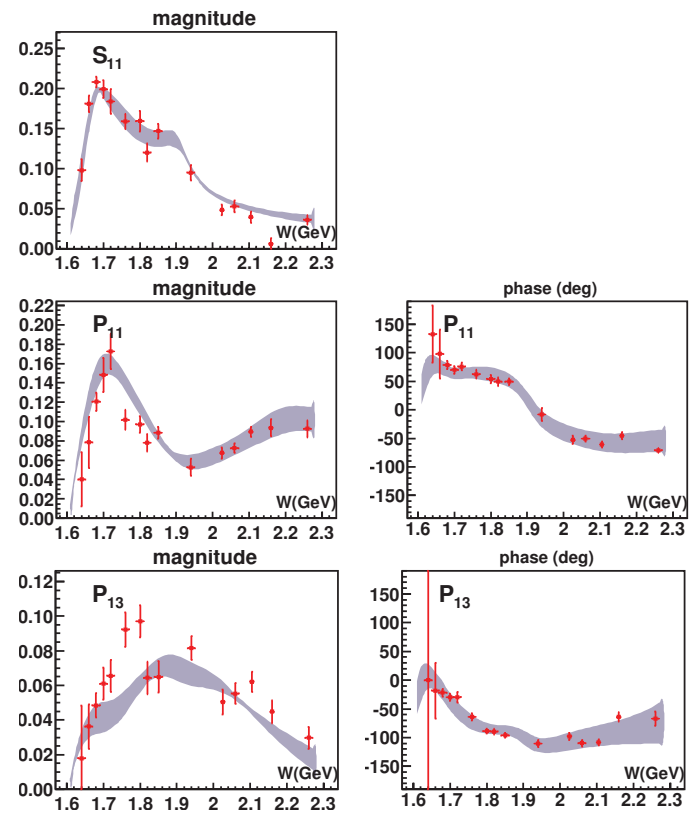

Figure 23. Fit with free $S$ and $P$-waves and $D, F$, and $G$-waves fixed to the energy-dependent solution BnGa2011-02 for $\gamma p \rightarrow$ $K^{+} \Lambda$, see [170] 
magnitude[mfm]

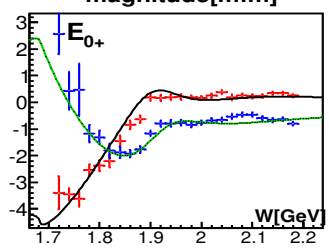

magnitude $[\mathrm{mfm}]$

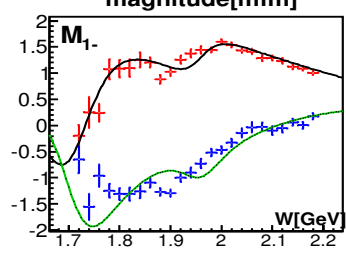

magnitude[mfm]

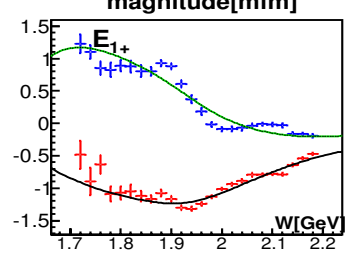

magnitude $[\mathrm{mfm}]$

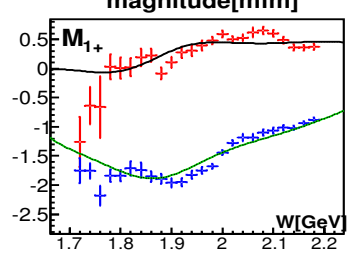

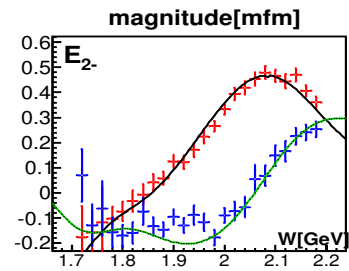

magnitude $[\mathrm{mfm}]$

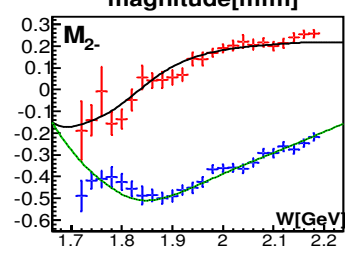

magnitude[mfm]

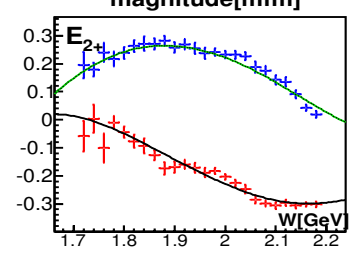

Figure 24. Preliminary results: real (red) and imaginary (blue) part of multipoles derived from a fit to data in slices of energy. The left column of subfigures is obtained without any constraints, for the multipoles in the right column, a penalty function is used. The lines show the energy-dependent Bonn-Gatchina fit. The results shown here were not included in the fit.

$P_{13}$ were obtained when the higher multipoles were fixed to the BnGa PWA solution (see Fig. 23). A first attempt to determine photoproduction multipoles for $\gamma p \rightarrow$ $K^{+} \Lambda$ was made in [170]. However, new precise data became available recently [171]. They allowed for a much more precise reconstruction of the multipoles. In the new analysis [172], the four largest multipoles $E_{0+}$, $M_{1^{-}}, E_{1+}$, and $M_{1+}$ were left free completely, the next three multipoles $E_{2-}, M_{2-}$, and $E_{2+}$ were "persuaded" by a penalty function to stay close to the BnGa energydependent fit (see Fig. 24). The multipoles are presently fitted applying the Laurent-Pittarinen expansion, a technique developed at Zagreb [173-175]. The preliminary results confirm with a model-independent method the existence of $N(1895) 1 / 2^{-}, N(1880) 1 / 2^{+}$, and $N(1900) 3 / 2^{+}$ whose existence must now be considered as established; $N(1875) 3 / 2^{-}$is strongly supported.

\section{Outlook}

One of the most important topics in baryon spectroscopy is to identify the effective degrees of freedom and respective forces which determine the spectrum and properties of baryon resonances. This includes answering the question if the missing resonances are proof of a quark-diquark structure of baryon excitations. The answer to the latter question is mostly settled thanks to the efforts to collect a large amount of high-quality data (differential cross sections and single and double polarization observables) for a large variety of different reactions. The numerosity of observed resonances excludes quasi-stable diquarks as part of the dynamics of excited baryons.

Some of resonances used to argue in this paper are not yet considered to be established ( $\geq 3$-star-resonances). However, further data are still in the pipe line or will be taken in future; higher statistic data and data on new polarisation observables will be obtained. Hopefully, the few still missing resonances (below $\sim 2.2 \mathrm{GeV}$ ) will be discovered in these forthcoming data. When the data on $\gamma p \rightarrow \pi^{+} \pi^{-} p$ are included in the PWA data base, practically all possible decay modes are included, and the sum of all branching ratios has to be close to 1 . When this condition is not fulfilled for a particular resonance, then that resonance should be considered with caution.

One class of baryon resonances has, however, so far escaped discovery: the lowest mass 20plet in Fig. 11 remains empty. A resonance $\mathrm{X}$ in the 20plet has an totally antisymmetric spin-flavor wave function. In quark models, these states have spacial wave functions in which both oscillators are excited simultaneously $\ell_{\rho}=\ell_{\lambda}=1$ and $\left|\vec{L}_{t o t}\right|=\left|\vec{\ell}_{\rho}+\vec{\ell}_{\lambda}\right|=1$.

High-mass nucleon resonances can have wave functions with a component in which both oscillators are excited. In section 3.2 we have argued that this component decays into an excited state plus meson, and not into the ground state plus meson. This observation implies that the resonance $\mathrm{X}$ in the lowest-mass 20plet cannot be excited from the ground state. Possibly, X can be seen only in a double cascade. In a first step, a high-mass resonance $\mathrm{R}$ is excited, in a second step, the high-mass resonance deexcites into a member of the 20plet, which then decays in a two-step cascade into ground state plus two mesons. This scenario demands a partial wave analysis of four-body final states. The decay sequences might be

$$
\gamma p \rightarrow R \rightarrow X+\pi \rightarrow N^{*} \pi+\pi \rightarrow N \pi \pi+\pi .
$$

The main problem is likely the combinatorial background.

An alternative approach is to search for the 20plet in the spectrum of $\Lambda$ excitations. The 20plet reduces to a spin-1/2 octet and a spin-3/2 singlet. One might search for a cascade

$$
\begin{aligned}
\gamma p \rightarrow R & \rightarrow \Lambda(x x x) J^{P}+K^{+} \\
& \rightarrow N^{*} K^{0}+K^{+} \rightarrow N \pi K^{0}+K^{+} .
\end{aligned}
$$

Expected are three states with $J^{P}=1 / 2^{+}, 3 / 2^{+}, 5 / 2^{+}$, and a $J^{P}=1 / 2^{+}, 3 / 2^{+}$doublet. Their preferential decay modes should be two-step cascades. The lowest mass resonance belongs to a spin- $1 / 2$ octet and is predicted to have a mass of $1747 \mathrm{MeV}$ [7]. Cascades like in (6) are energetically disfavored and thus, $\Lambda(1747) 1 / 2^{+}$might be narrow.

Of course, one has to keep in mind that our argument given in section 3.2, that wave function components where both oscillators are excited simultaneously decay into an 
excited state plus meson and not into the ground state plus meson was deduced from the observation of different $p 2 \pi$ decay modes of $N^{*}$ - and $\Delta^{*}$-resonances which individually still have large error bars. New high statistics $\gamma p \rightarrow p \pi^{+} \pi^{-}$ data, as well as new $\gamma p \rightarrow p 2 \pi^{0}$ data taken with polarized beam and polarized target are presently included in our data base. These data will provide further contraints to more firmly determine these decay modes.

The number of resonances could, of course, also be higher than quark models predict. Theories which generate baryons from meson-baryon interactions have made great success in reproducing precisely properties of some baryon resonances. So far they fail, however, to generate the full spectrum, and the relation between quark-model states and dynamically generated resonances is still unclear. Intriguing are poles like $N(1750) 1 / 2^{+}$[54] which are dynamically generated and not seen in other PWAs. Is this a genuine resonance? Then it should be seen as well in phenomenological coupled-channel analyses. More precise data will provide the potential for new discoveries, a more precise determination of the photoproduction multipoles and a better definition of branching ratios.

$N^{*}$-resonances may strongly couple to the neutron but they may only weekly or not at all couple to the proton. Therefore resonances, maybe even resonances of specific symmetries or nature, may escape detection if only proton data is analyzed. This bias can be overcome if also photoproduction data off the neutron, which is presently still scarce, is investigated. A model which predicts e.g. resonances with a large photocoupling for production off neutrons and a small coupling for production off protons is the chiral soliton model [142, 143].

One of the last missing degrees of freedom in the experiments is the isospin dependence since the electromagnetic interaction does not conserve isospin. The electromagnetic transition operator can be split into an isovector and an isoscalar part, giving rise to three independent matrix elements [176]. Photoproduction of isovector mesons, such as the pions, involve all three matrix elements, while in the case of isoscalar meson photoproduction only two contribute. To study the isospin structure of the amplitudes, photoproduction experiments on the neutron are unavoidable. This becomes directly obvious in the isoscalar meson production. It is hence important to study photoproduction off neutrons with the same precision as achieved for protons.

Topical will also be the search for baryon resonances with a large hybrid content. Dudek and Edwards calculated the hybrid contribution to the wave functions of baryon resonances and found that are are a few distinctive resonances in which the gluon field is excited [21]. Ordinary baryons and hybrid baryons show a different $Q^{2}$ dependence in electroproduction. In quark model calculations, the transverse helicity amplitude for a hybrid state decreases much faster than that for a radially excited state; in addition, the hybrid longitudinal helicity amplitude vanishes [31, 32]. Identification of hybrid contributions in high-mass baryon resonances is certainly a most challenging and rewarding task for future experimental and theoretical studies. Electroproduction of baryon resonances provides the necessary information to disentangle the $q q q$ component in the baryonic wave function, the meson-baryon cloud contributions and a potential hybrid component. The latter two contributions are expected to contribute especially at low $Q^{2}$.

Summarizing, we have seen that a number of important questions in baryon spectroscopy has found an answer. At the same time, new questions have emerged. To answer these questions, more precise data are required. Here, data on polarization observables will play a most significant role.

We appreciate the constructive atmosphere within the SFB/TR16 and would like to thank all SFB members, professors, post-docs, students, technicians, and administrative assistents for the fruitful cooperation. In particular, we would like to mention the contributions of V. Nikonov and A. Anisovich to this project. The work reported here would not have been possible without the support from the Deutsche Forschungsgemeinschaft within the SFB/TR16.

\section{References}

[1] N. Isgur, "Why $N *$ 's are important," nucl-th/0007008, JLAB-THY-00-20; in: NSTAR2000: Excited Nucleons and Hadronic Structure, Feb. 16-19, 2000, Newport News, Virginia. Proceedings: N. Isgur, V.D. Burkert et al. (eds.), Singapore, World Scientific, 2001.

[2] E. Klempt and J.M. Richard, "Baryon spectroscopy," Rev. Mod. Phys. 82, 1095 (2010).

[3] V. Crede and W. Roberts, "Progress towards understanding baryon resonances," Rept. Prog. Phys. 76, 076301 (2013).

[4] L.Y. Glozman, W. Plessas, K. Varga and R.F. Wagenbrunn, "Unified description of light and strange baryon spectra," Phys. Rev. D 58, 094030 (1998).

[5] S. Capstick and W. Roberts, "Quark models of baryon masses and decays", Prog. Part. Nucl. Phys. 45, S241 (2000).

[6] U.Löring, B.C. Metsch and H.R. Petry, "The light baryon spectrum in a relativistic quark model with instanton-induced quark forces: The non-strange baryon spectrum and ground-states", Eur. Phys. J. A 10, 395 (2001).

[7] U. Löring, B.C. Metsch and H.R. Petry, "The light baryon spectrum in a relativistic quark model with instanton induced quark forces: The strange baryon spectrum", Eur. Phys. J. A 10, 447 (2001).

[8] M. Ronniger and B.C. Metsch, "Effects of a spinflavour dependent interaction on the baryon mass spectrum,” Eur. Phys. J. A 47, 162 (2011).

[9] E. Santopinto and J. Ferretti, "Strange and nonstrange baryon spectra in the relativistic interacting quarkdiquark model with a Gürsey and Radicati-inspired exchange interaction," Phys. Rev. C 92, 025202 (2015).

[10] R.G. Edwards, J.J. Dudek, D.G. Richards and S.J. Wallace, "Excited state baryon spectroscopy from lattice QCD”, Phys. Rev. D 84, 074508 (2011). 
[11] G. Höhler, F. Kaiser, R. Koch and E. Pietarinen, "Handbook Of Pion Nucleon Scattering", Published by Fachinform. Zentr. Karlsruhe 1979, 440 P. (Physics Data, No.12-1 (1979)).

[12] R.E. Cutkosky, C.P. Forsyth, J.B. Babcock, R.L. Kelly and R.E. Hendrick, "Pion - Nucleon Partial Wave Analysis", 4th Int. Conf. on Baryon Resonances, Toronto, Canada, Jul 14-16, 1980. Published in Baryon 1980:19 (QCD161:C45:1980).

[13] R.A. Arndt, Z.-J. Li, L.D. Roper, R.L. Workman and J.M. Ford, "Pion - nucleon partial wave analysis to 2 GeV”, Phys. Rev. D 43, 2131 (1991).

[14] D.E. Groom et al. [Particle Data Group Collaboration], "Review of Particle Physics. Particle Data Group,’ Eur. Phys. J. C 15, 1 (2000).

[15] R.A. Arndt, W.J. Briscoe, I.I. Strakovsky and R.L. Workman, "Extended partial-wave analysis of $\pi N$ scattering data", Phys. Rev. C 74, 045205 (2006).

[16] I.G. Alekseev et al., "Influence of spin-rotation measurements on partial-wave analyses of elastic pion nucleon scattering", Phys. Rev. C 55, 2049 (1997).

[17] I.G. Alekseev et al., "Measurements of spin rotation parameter $\mathrm{A}$ in pion proton elastic scattering at 1.62GeV/c", Phys. Lett. B 485, 32 (2000).

[18] I.G. Alekseev et al., "Measurement of the spin rotation parameter $\mathrm{A}$ in the elastic pion proton scattering at 1.43-GeV/c", Eur. Phys. J. C 45, 383 (2006).

[19] I.G. Alekseev et al., "Backward asymmetry measurements in the elastic pion-proton scattering at resonance energies", Eur. Phys. J. A 39, 163 (2009).

[20] S. Capstick and P.R. Page, "Hybrid and conventional baryons in the flux tube model," Phys. Rev. C 66, 065204 (2002).

[21] J.J. Dudek and R.G. Edwards, "Hybrid baryons in QCD,” Phys. Rev. D 85, 054016 (2012).

[22] M. Anselmino, E. Predazzi, S. Ekelin, S. Fredriksson and D.B. Lichtenberg, "Diquarks," Rev. Mod. Phys. 65, 1199 (1993).

[23] M. Kirchbach, M. Moshinsky and Y. F. Smirnov, "Baryons in O(4) and vibron model," Phys. Rev. D 64, 114005 (2001).

[24] R.L. Jaffe and F. Wilczek, "Diquarks and exotic spectroscopy,” Phys. Rev. Lett. 91, 232003 (2003).

[25] R.L. Jaffe, “Exotica," Phys. Rept. 409, 1 (2005).

[26] E. Santopinto, "An interacting quark-diquark model of baryons,” Phys. Rev. C 72, 022201 (2005).

[27] S. Eidelman et al. [Particle Data Group Collaboration], "Review of Particle Physics. Particle Data Group,” Phys. Lett. B 592, 1 (2004).

[28] R. Koniuk, N. Isgur, "Where have all the resonances gone? An analysis of baryon couplings in a quark model with chromodynamics", Phys. Rev. Lett. 44, 845 (1980).

[29] H.P. Morsch and P. Zupranski, "Structure of the $P_{11}$ (1440) resonance from $\alpha p$ and $\pi N$ scattering," Phys. Rev. C 61, 024002 (2000).

[30] A. V. Sarantsev et al., "New results on the Roper resonance and the $P_{11}$ partial wave," Phys. Lett. B 659, 94
(2008).

[31] Z. P. Li, "Photoproduction signatures of hybrid baryons: an application of the quark model with gluonic degrees of freedom," Phys. Rev. D 44, 2841 (1991).

[32] Z.P. Li, V. Burkert and Z.J. Li, "Electroproduction of the Roper resonance as a hybrid state," Phys. Rev. D 46, 70 (1992).

[33] S. Schneider, S. Krewald and U.-G. Meissner, "The reaction $\pi N \rightarrow \pi \pi N$ in a meson-exchange approach," Eur. Phys. J. A 28, 107 (2006).

[34] I.G. Aznauryan et al. [CLAS Collaboration], "Electroexcitation of nucleon resonances from CLAS data on single pion electroproduction," Phys. Rev. C 80, 055203 (2009) .

[35] R.H. Dalitz, "K-nucleon bound-state interpretation of the $1385 \mathrm{MeV} \pi-\Lambda$ resonance," Phys. Rev. Lett. 6, 239 (1961).

[36] L.Y. Glozman, "Restoration of chiral and U(1)A symmetries in excited hadrons," Phys. Rept. 444, 1 (2007).

[37] L.Y. Glozman, "Chiral multiplets of excited mesons," Phys. Lett. B 587, 69 (2004).

[38] L.Y. Glozman, "Parity doublets and chiral symmetry restoration in baryon spectrum," Phys. Lett. B 475, 329 (2000).

[39] W.J. Briscoe et al., gwdac.phys. gwu.edu/.

[40] A. Anisovich, E. Klempt, A. Sarantsev and U. Thoma, "Partial wave decomposition of pion and photoproduction amplitudes”, Eur. Phys. J. A 24, 111 (2005).

[41] E. Klempt, A.V. Anisovich, V.A. Nikonov, A.V. Sarantsev and U. Thoma, "Phase motion of baryon resonances", Eur. Phys. J. A 29, 307 (2006).

[42] A.V. Anisovich and A.V. Sarantsev, "Partial decay widths of baryons in the spin-momentum operator expansion method", Eur. Phys. J. A 30, 427 (2006).

[43] A.V. Anisovich, V.V. Anisovich, E. Klempt, V.A. Nikonov and A.V. Sarantsev, "Baryon-baryon and baryon-antibaryon interaction amplitudes in the spinmomentum operator expansion method," Eur. Phys. J. A 34, 129 (2007).

[44] A.V. Anisovich, A. Sarantsev, O. Bartholomy, E. Klempt, V.A. Nikonov and U. Thoma, "Photoproduction of baryons decaying into $N \pi$ and $N \eta$ ", Eur. Phys. J. A 25, 427 (2005).

[45] A.V. Anisovich, V. Kleber, E. Klempt, V.A. Nikonov, A.V. Sarantsev and U. Thoma, "Baryon resonances and polarization transfer in hyperon photoproduction", Eur. Phys. J. A 34, 423 (2007).

[46] A.V. Anisovich, E. Klempt, V.A. Nikonov, M.A. Matveev, A.V. Sarantsev and U. Thoma, "Photoproduction of pions and properties of baryon resonances from a Bonn-Gatchina partial wave analysis," Eur. Phys. J. A 44, 203 (2010).

[47] A.V. Anisovich, E. Klempt, V.A. Nikonov, A.V. Sarantsev and U. Thoma, "P-wave excited baryons from pion- and photo-induced hyperon 
production”, Eur. Phys. J. A 47, 27 (2011).

[48] A.V. Anisovich, E.Klempt, V.A. Nikonov, A.V. Sarantsev and U. Thoma, "Nucleon resonances in the fourth resonance region", Eur. Phys. J. A 47, 153 (2011).

[49] A. V. Anisovich, R. Beck, E. Klempt, V. A. Nikonov, A. V. Sarantsev and U. Thoma, "Properties of baryon resonances from a multichannel partial wave analysis", Eur. Phys. J. A 48, 15 (2012).

[50] T. Sato and T.-S.H. Lee, "Dynamical Models of the Excitations of Nucleon Resonances", J. Phys. G G 36, 073001 (2009).

[51] M. Döring, C. Hanhart, F. Huang, S. Krewald and U.-G. Meißner, "The Role of the background in the extraction of resonance contributions from meson-baryon scattering", Phys. Lett. B 681, 26 (2009).

[52] M. Döring, C. Hanhart, F. Huang, S. Krewald and U.G. Meißner, "Analytic properties of the scattering amplitude and resonances parameters in a meson exchange model", Nucl. Phys. A 829, 170 (2009).

[53] M. Döring, C. Hanhart, F. Huang, S. Krewald, U.G. Meissner and D. Rönchen, "The reaction $\pi^{+} p \rightarrow$ $K^{+} \Sigma^{+}$in a unitary coupled-channels model," Nucl. Phys. A 851, 58 (2011).

[54] D. Rönchen et al., "Coupled-channel dynamics in the reactions $\pi N \rightarrow \pi N, \eta N, K \Lambda, K \Sigma$,” Eur. Phys. J. A 49, 44 (2013).

[55] D. Rönchen et al., "Photocouplings at the Pole from Pion Photoproduction,” Eur. Phys. J. A 50, 101 (2014) Erratum: [Eur. Phys. J. A 51, 63 (2015)].

[56] D. Rönchen, M. Döring, H. Haberzettl, J. Haidenbauer, U.-G. Meißner and K. Nakayama, "Eta photoproduction in a combined analysis of pion- and photoninduced reactions," Eur. Phys. J. A 51, 70 (2015).

[57] G.Y. Chen, S.S. Kamalov, S.N. Yang, D. Drechsel and L. Tiator, "Nucleon resonances in $\pi N$ scattering up to energies $s^{1 / 2}<2.0-\mathrm{GeV}$ ", Phys. Rev. C 76, 035206 (2007).

[58] A. Matsuyama, T. Sato and T. -S. H. Lee, "Dynamical coupled-channel model of meson production reactions in the nucleon resonance region", Phys. Rept. 439, 193 (2007).

[59] S. Sarkar, E. Oset and M.J. Vicente Vacas, "Baryonic resonances from baryon decuplet-meson octet interaction", Nucl. Phys. A 750, 294 (2005). [Erratum-ibid. A 780 (2006) 78].

[60] G. Penner and U. Mosel, "Vector meson production and nucleon resonance analysis in a coupled channel approach for energies $m_{N}<s^{1 / 2}<2-\mathrm{GeV}$. 1. Pion induced results and hadronic parameters", Phys. Rev. C 66, 055211 (2002).

[61] G. Penner and U. Mosel, "Vector meson production and nucleon resonance analysis in a coupled channel approach for energies $m_{N}<s^{1 / 2}<2-\mathrm{GeV}$. 2. Photon induced results," Phys. Rev. C 66, 055212 (2002).

[62] I. Denisenko et al., " $N *$ decays to $N \omega$ from new data on $\gamma p \rightarrow \omega p$, , Phys. Lett. B 755, 97 (2016).
[63] D.M. Manley and E.M. Saleski, "Multichannel resonance parametrization of $\pi N$ scattering amplitudes", Phys. Rev. D 45, 4002 (1992).

[64] R.A. Arndt, W.J. Briscoe, I.I. Strakovsky, R.L. Workman and M.M. Pavan, "Dispersion relation constrained partial wave analysis of $\pi N$ elastic and $\pi N \rightarrow \eta N$ scattering data: The Baryon spectrum", Phys. Rev. C 69, 035213 (2004).

[65] V. Crede et al. [CB-ELSA Collaboration], "Photoproduction of $\eta$ mesons off protons for $0.75 \mathrm{GeV}<$ $E_{\gamma}<3$ GeV," Phys. Rev. Lett. 94, 012004 (2005).

[66] H. van Pee et al. [CB-ELSA Collaboration], "Photoproduction of $\pi^{0}$-mesons off protons from the $\Delta(1232)$ region to $E_{\gamma}=3 \mathrm{GeV}$," Eur. Phys. J. A 31, 61 (2007).

[67] O. Bartholomy et al. [CB-ELSA Collaboration], "Neutral pion photoproduction off protons in the energy range $0.3 \mathrm{GeV}<E_{\gamma}<3 \mathrm{GeV}$," Phys. Rev. Lett. 94, 012003 (2005).

[68] O. Bartholomy et al. [CB-ELSA Collaboration], "Photoproduction of $\eta$-mesons off protons," Eur. Phys. J. A 33, 133 (2007).

[69] A.V. Sarantsev, V.A. Nikonov, A.V. Anisovich, E. Klempt and U. Thoma, "Decays of baryon resonances into $\Lambda K^{+}, \Sigma^{0} K^{+}$and $\Sigma^{+} K^{0}$," Eur. Phys. J. A 25, 441 (2005).

[70] U. Thoma et al., " $N^{*}$ and $\Delta^{*}$ decays into $N \pi^{0} \pi^{0}$," Phys. Lett. B 659, 87 (2008).

[71] J. Beringer et al. [Particle Data Group Collaboration], "Review of Particle Physics (RPP)," Phys. Rev. D 86, 010001 (2012).

[72] R. K. Bradford et al. [CLAS Collaboration], "First measurement of beam-recoil observables $\mathrm{C}(\mathrm{x})$ and $\mathrm{C}(\mathrm{z})$ in hyperon photoproduction," Phys. Rev. C 75, 035205 (2007).

[73] V.A. Nikonov, A.V. Anisovich, E.Klempt, A.V. Sarantsev and U. Thoma, "Further evidence for N(1900) P(13) from photoproduction of hyperons," Phys. Lett. B 662, 245 (2008).

[74] V. Sokhoyan et al. [CBELSA/TAPS Collaboration], "High-statistics study of the reaction $\gamma p \rightarrow p 2 \pi^{0}$," Eur. Phys. J. A 51, 95 (2015); Erratum: [Eur. Phys. J. A 51, no. 12, 187 (2015)].

[75] E. Gutz et al. [CBELSA and TAPS Collaborations], "Photoproduction of meson pairs: First measurement of the polarization observable $I^{* *} s$," Phys. Lett. B 687, 11 (2010).

[76] E. Gutz et al. [CBELSA/TAPS Collaboration], "High statistics study of the reaction $\gamma p \rightarrow p \pi^{0} \eta$," Eur. Phys. J. A 50, 74 (2014).

[77] Y. Assafiri et al., "Double $\pi^{0}$ photoproduction on the proton at GRAAL," Phys. Rev. Lett. 90, 222001 (2003).

[78] V.L. Kashevarov et al., "Study of the $\gamma p \rightarrow \pi^{0} \pi^{0} p$ reaction with the Crystal Ball/TAPS at the Mainz," Phys. Rev. C 85, 064610 (2012).

[79] J.R.M. Annand et al. [A2 Collaboration], "First measurement of target and beam-target asymmetries in the $\gamma p \rightarrow \pi^{0} \eta p$ reaction," Phys. Rev. C 91, 055208 (2015). 
[80] V. Sokhoyan et al. [CBELSA/TAPS Collaboration], "Data on $I^{s}$ and $I^{c}$ in $\vec{\gamma} p \rightarrow p \pi^{0} \pi^{0}$ reveal cascade decays of $N(1900)$ via $N(1520) \pi$," Phys. Lett. B 746, 127 (2015).

[81] A. Thiel et al. [CBELSA/TAPS Collaboration], "Three-body nature of $N^{*}$ and $\Delta^{*}$ resonances from sequential decay chains," Phys. Rev. Lett. 114, 091803 (2015).

[82] J. Müller et al. [CBELSA/TAPS Collaboration], "New data on $\vec{\gamma} \vec{p} \rightarrow \eta p$ with polarized photons and protons and their implications for $N^{*} \rightarrow N \eta$ decays", submitted to Physics Letters B.

[83] C.S. Akondi et al., "Measurement of the transverse target and beam-target asymmetries in $\eta$ meson photoproduction at MAMI," Phys. Rev. Lett. 113, 102001 (2014).

[84] I. Senderovich et al. [CLAS Collaboration], "First measurement of the helicity asymmetry $E$ in $\eta$ photoproduction on the proton," Phys. Lett. B 755, 64 (2016).

[85] V. Crede et al. [CBELSA/TAPS Collaboration], "Photoproduction of $\eta$ and $\eta^{\prime}$ mesons off protons," Phys. Rev. C 80, 055202 (2009).

[86] B. Krusche et al., Prog. Part. Nucl. Phys. 51, 399 (2003).

[87] K.A. Olive et al. [Particle Data Group Collaboration], "Review of Particle Physics," Chin. Phys. C 38, 090001 (2014).

[88] A.V. Anisovich, E. Klempt, B. Krusche, V.A. Nikonov, A.V. Sarantsev, U. Thoma and D. Werthmüller, "Interference phenomena in the $J^{P}=1 / 2^{-}$wave in $\eta$ photoproduction," Eur. Phys. J. A 51, 72 (2015).

[89] I. Jaegle et al. [CBELSA/TAPS Collaboration], "Photoproduction of $\eta^{\prime}$-mesons off the deuteron," Eur. Phys. J. A 47, 11 (2011).

[90] A.V. Anisovich, E. Klempt, V.A. Nikonov, A.V. Sarantsev, H. Schmieden and U. Thoma, "Evidence for a negative-parity spin-doublet of nucleon resonances at $1.88 \mathrm{GeV}$," Phys. Lett. B 711, 162 (2012).

[91] R. Castelijns et al. [CBELSA/TAPS Collaboration], "Nucleon resonance decay by the $K^{0} \Sigma^{+}$channel," Eur. Phys. J. A 35, 39 (2008).

[92] R. Ewald et al. [CBELSA/TAPS Collaboration], "Anomaly in the $K_{S}^{0} \Sigma^{+}$photoproduction cross section off the proton at the $K^{*}$ threshold," Phys. Lett. B 713, 180 (2012).

[93] R. Ewald et al. [CBELSA/TAPS Collaboration], "Measurement of polarisation observables in $K_{s}^{0} \Sigma^{+}$ photoproduction off the proton," Phys. Lett. B 738, 268 (2014).

[94] D. Elsner et al. [CBELSA and TAPS Collaborations], "Measurement of the beam asymmetry in $\eta$ photoproduction off the proton," Eur. Phys. J. A 33, 147 (2007).

[95] N. Sparks et al. [CBELSA/TAPS Collaboration], "Measurement of the Beam Asymmetry $\Sigma$ in the Forward Direction for $\vec{\gamma} p \rightarrow p \pi^{0}$ Photoproduction," Phys.
Rev. C 81, 065210 (2010).

[96] K. Nakamura et al. [Particle Data Group Collaboration], "Review of particle physics," J. Phys. G 37, 075021 (2010).

[97] E. Klempt and B.C. Metsch, "Multiplet classification of light-quark baryons," Eur. Phys. J. A 48, 127 (2012).

[98] M. Shrestha and D.M. Manley, "Multichannel parametrization of $\pi N$ scattering amplitudes and extraction of resonance parameters," Phys. Rev. C 86, 055203 (2012).

[99] O. V. Maxwell, "Electromagnetic production of kaons from protons, and baryon electromagnetic form factors," Phys. Rev. C 85, 034611 (2012).

[100] T. Mart and M.J. Kholili, "Origin of the second peak in the cross section of the $K^{+} \Lambda$ photoproduction," Phys. Rev. C 86, 022201 (2012)

[101] V. Shklyar, H. Lenske and U. Mosel, " $\eta$-meson production in the resonance-energy region," Phys. Rev. C 87, 015201 (2013).

[102] A.V. Anisovich, E. Klempt, V.A. Nikonov, A.V. Sarantsev and U. Thoma, "Evidence for a spinquartet of nucleon resonances at $2 \mathrm{GeV}$," Phys. Lett. B 711, 167 (2012).

[103] A.V. Anisovich, V. Burkert, E. Klempt, V.A. Nikonov, A.V. Sarantsev and U. Thoma, "Helicity amplitudes for photoexcitation of nucleon resonances off neutrons," Eur. Phys. J. A 49, 67 (2013).

[104] A.V. Anisovich et al., "Neutron helicity amplitudes", in preparation.

[105] N. Matagne and F. Stancu, "Updated $1 / N_{c}$ expansion analysis of $\left[\mathbf{5 6}, \mathbf{2}^{+}\right]$and $\left[\mathbf{7 0}, \ell^{+}\right]$baryon multiplets," Phys. Rev. D 93, 096004 (2016).

[106] S. Weinberg, "Phenomenological Lagrangians," Physica A 96, 327 (1979).

[107] M. Gell-Mann, R. J. Oakes and B. Renner, "Behavior of current divergences under SU(3) x SU(3)," Phys. Rev. 175, 2195 (1968).

[108] Y. Nambu, "Quasiparticles and Gauge Invariance in the Theory of Superconductivity," Phys. Rev. 117, 648 (1960).

[109] J. Goldstone, "Field theories with superconductor solutions," Nuovo Cim. 19, 154 (1961).

[110] I. Horn et al. [CB-ELSA Collaboration], "Evidence for a parity doublet $\Delta(1920) P_{33}$ and $\Delta(1940) D_{33}$ from $\gamma p \rightarrow p \pi^{0} \eta$,' Phys. Rev. Lett. 101, 202002 (2008).

[111] I. Horn et al. [CB-ELSA Collaboration], "Study of the reaction $\gamma p \rightarrow p \pi^{0} \eta$, , Eur. Phys. J. A 38, 173 (2008).

[112] L. Y. Glozman and A. V. Nefediev, "Chiral restoration in excited nucleons versus SU(6)," Nucl. Phys. A 807, 38 (2008).

[113] E. Klempt and A. Zaitsev, "Glueballs, Hybrids, Multiquarks. Experimental facts versus QCD inspired concepts," Phys. Rept. 454, 1 (2007).

[114] L.Y. Glozman, private communication to E.K.

[115] J. Hartmann et al., "The $N(1520) 3 / 2^{+}$helicity amplitudes from an energy-independent multipole analysis based on new polarization data on photoproduction of 
neutral pions," Phys. Rev. Lett. 113, 062001 (2014).

[116] J. Hartmann et al. [CBELSA/TAPS Collaboration], "The polarization observables T, $\mathrm{P}$, and $\mathrm{H}$ and their impact on $\gamma p \rightarrow p \pi^{0}$ multipoles," Phys. Lett. B 748, 212 (2015).

[117] M. Gottschall et al. [CBELSA/TAPS Collaboration], "First measurement of the helicity asymmetry for $\gamma p \rightarrow p \pi^{0}$ in the resonance region," Phys. Rev. Lett. 112, 012003 (2014).

[118] A. Thiel et al., "Double-polarization observable G in neutral-pion photoproduction off the proton," submitted to EPJA, arXiv:1604.02922 [nucl-ex].

[119] S. Strauch et al. [CLAS Collaboration], "First Measurement of the Polarization Observable $\mathrm{E}$ in the $\vec{p}\left(\vec{\gamma}, \pi^{+}\right) n$ Reaction up to $2.25 \mathrm{GeV}$,' Phys. Lett. B 750, 53 (2015).

[120] E. Klempt, private communication.

[121] A. V. Anisovich, V. Burkert, E. Klempt, V. A. Nikonov, E. Pasyuk, A. V. Sarantsev, S. Strauch and U. Thoma, "Existence of $\Delta(2200) 7 / 2^{-}$precludes chiral symmetry restoration at high mass," arXiv:1503.05774 [nucl-ex].

[122] E. Klempt, "A Mass formula for baryon resonances,” Phys. Rev. C 66, 058201 (2002).

[123] H. Forkel and E. Klempt, "Diquark correlations in baryon spectroscopy and holographic QCD," Phys. Lett. B 679, 77 (2009).

[124] E. Klempt, "Nucleon Excitations," Chin. Phys. C 34, 1241 (2010).

[125] E. Klempt, "Do parity doublets in the baryon spectrum reflect restoration of chiral symmetry?," Phys. Lett. B 559, 144 (2003).

[126] E. Klempt, "Delta resonances, Quark models, chiral symmetry and AdS/QCD," Eur. Phys. J. A 38, 187 (2008).

[127] G. Wolf, "Review of High Energy Diffraction in Real and Virtual Photon Proton scattering at HERA," Rept. Prog. Phys. 73, 116202 (2010).

[128] A. Donnachie and P. V. Landshoff, "Exclusive vector meson production at HERA,” Phys. Lett. B 348, 213 (1995).

[129] A. Donnachie and P. V. Landshoff, "Exclusive vector photoproduction: Confirmation of Regge theory," Phys. Lett. B 478, 146 (2000).

[130] J. Barth et al., "Low-energy of photoproduciton of $\omega$-mesons,” Eur. Phys. J. A 18, 117 (2003).

[131] J. Ajaka et al. [GRAAL Collaboration], "Evidence for nucleon-resonance excitation in $\omega$-meson photoproduction," Phys. Rev. Lett. 96, 132003 (2006).

[132] F. Klein et al. [CBELSA/TAPS Collaboration], "Beam asymmetry in near threshold $\omega$ photoproduction off the proton," Phys. Rev. D 78, 117101 (2008).

[133] M. Williams et al. [CLAS Collaboration], "Differential cross sections and spin density matrix elements for the reaction $\gamma p \rightarrow p \omega$," Phys. Rev. C 80, 065208 (2009).

[134] M. Williams et al. [CLAS Collaboration], "Partial wave analysis of the reaction $\gamma p \rightarrow p \omega$ and the search for nucleon resonances," Phys. Rev. C 80, 065209 (2009).

[135] I.I. Strakovsky et al., "Photoproduction of the $\omega$ meson on the proton near threshold," Phys. Rev. C 91, 045207 (2015) .

[136] A. Wilson et al. [CBELSA/TAPS Collaboration], "Photoproduction of $\omega$ mesons off the proton," Phys. Lett. B 749, 407 (2015).

[137] H. Eberhardt et al., "Measurement of double polarisation asymmetries in $\omega$-photoproduction," Phys. Lett. B 750, 453 (2015).

[138] F. Dietz et al. [CBELSA/TAPS Collaboration], "Photoproduction of $\omega$ mesons off protons and neutrons,” Eur. Phys. J. A 51, 6 (2015).

[139] J. Junkersfeld et al. [CB-ELSA Collaboration], "Photoproduction of $\pi^{0} \omega$ off protons for $E_{\gamma} \leq 3 \mathrm{GeV}$," Eur. Phys. J. A 31, 365 (2007).

[140] A.V. Anisovich et al. [CLAS Collaboration], "Differential cross sections and polarization observables from the CLAS $K^{*}$ photoproduction and search for new $N^{*}$ states", in collaboration review.

[141] M. Nanova et al. [CBELSA/TAPS Collaboration], " $K^{0} \pi^{0} \Sigma^{+}$and $K^{* 0} \Sigma^{+}$photoproduction off the proton," Eur. Phys. J. A 35, 333 (2008).

[142] D. Diakonov, V. Petrov and M.V. Polyakov, "Exotic anti-decuplet of baryons: Prediction from chiral solitons," Z. Phys. A 359, 305 (1997).

[143] M.V. Polyakov and A. Rathke, "On photoexcitation of baryon anti-decuplet," Eur. Phys. J. A 18, 691 (2003).

[144] V. Kuznetsov and M.V. Polyakov, "New Narrow Nucleon $N^{*}$ (1685)," JETP Lett. 88, 347 (2008).

[145] R.A. Arndt, Y.I. Azimov, M.V. Polyakov, I.I. Strakovsky and R.L. Workman, "Nonstrange and other unitarity partners of the exotic $\Theta^{+}$baryon," Phys. Rev. C 69, 035208 (2004).

[146] V. Kuznetsov et al. [GRAAL Collaboration], "Evidence for a narrow structure at $\mathrm{W} 1.68-\mathrm{GeV}$ in $\eta$ photoproduction on the neutron," Phys. Lett. B 647, 23 (2007).

[147] T. Liu, Y. Mao and B.Q. Ma, "Present status on experimental search for pentaquarks," Int. J. Mod. Phys. A 29, 1430020 (2014).

[148] I. Jaegle et al. [CBELSA and TAPS Collaborations], "Quasi-free photoproduction of $\eta$-mesons of the neutron,” Phys. Rev. Lett. 100, 252002 (2008).

[149] I. Jaegle et al., "Quasi-free photoproduction of $\eta$ mesons off the deuteron," Eur. Phys. J. A 47, 89 (2011).

[150] D. Werthmüller et al. [A2 Collaboration], "Narrow Structure in the Excitation Function of $\eta$ Photoproduction off the Neutron," Phys. Rev. Lett. 111, 232001 (2013).

[151] V. Shklyar, H. Lenske and U. Mosel, " $\eta$ photoproduction in the resonance energy region," Phys. Lett. B 650, 172 (2007).

[152] R. Shyam and O. Scholten, "Photoproduction of $\eta$ meson within a coupled-channels K-matrix approach," Phys. Rev. C 78, 065201 (2008). 
[153] M. Döring and K. Nakayama, "On the cross section ratio $\sigma(n) / \sigma(p)$ in $\eta$ photoproduction," Phys. Lett. B 683, 145 (2010).

[154] A.V. Anisovich, I. Jaegle, E. Klempt, B. Krusche, V.A. Nikonov, A.V. Sarantsev and U. Thoma, "Photoproduction of $\eta$ mesons off neutrons from a deuteron target," Eur. Phys. J. A 41, 13 (2009).

[155] A. V. Anisovich, E. Klempt, V. Kuznetsov, V. A. Nikonov, M. V. Polyakov, A. V. Sarantsev and U. Thoma, "Study of the narrow structure at $1685 \mathrm{MeV}$ in $\gamma p \rightarrow \eta p$," Phys. Lett. B 719, 89 (2013).

[156] X.H. Zhong and Q. Zhao, " $\eta$ photoproduction on the quasi-free nucleons in the chiral quark model," Phys. Rev. C 84, 045207 (2011).

[157] A. Fantini et al., "First measurement of the $\Sigma$ beam asymmetry in $\eta$ photoproduction on the neutron," Phys. Rev. C 78, 015203 (2008).

[158] R. Di Salvo et al., "Measurement of $\Sigma$ beam asymmetry in $\pi^{0}$ photoproduction off the neutron in the second and third resonances region," Eur. Phys. J. A 42, 151 (2009).

[159] A.V. Anisovich, E. Klempt, V.A. Nikonov, A.V. Sarantsev and U. Thoma, "Sign ambiguity in the K $\Sigma$ channel,” Eur. Phys. J. A 49, 158 (2013).

[160] A.V. Anisovich, R. Beck, E. Klempt, V.A. Nikonov, A.V. Sarantsev and U. Thoma, "Pion- and photoinduced transition amplitudes to $\Lambda K, \Sigma K$, and $N \eta$," Eur. Phys. J. A 48, 88 (2012).

[161] A.V. Anisovich et al., "The Impact of New Polarization Data from Bonn, Mainz and Jefferson Laboratory on $\gamma p \rightarrow \pi N$ Multipoles," Eur. Phys. J. A 52, 284 (2016).

[162] G.F. Chew, M.L. Goldberger, F.E. Low and Y. Nambu, "Relativistic dispersion relation approach to photomeson production," Phys. Rev. 106, 1345 (1957). [163]

[163] I.S. Barker, A. Donnachie and J K. Storrow, "Complete Experiments in Pseudoscalar Photoproduction," Nucl. Phys. B 95, 347 (1975).

[164] W.T. Chiang and F. Tabakin, "Completeness rules for spin observables in pseudoscalar meson photoproduction," Phys. Rev. C 55, 2054 (1997).

[165] A. M. Sandorfi, S. Hoblit, H. Kamano and T.S. H. Lee, "Determining pseudoscalar meson photo- production amplitudes from complete experiments," J. Phys. G 38, 053001 (2011).

[166] Y. Wunderlich, R. Beck and L. Tiator, "The complete-experiment problem of photoproduction of pseudoscalar mesons in a truncated partial-wave analysis,” Phys. Rev. C 89, 055203 (2014).

[167] R.L. Workman, M.W. Paris, W.J. Briscoe and I.I. Strakovsky, "Unified Chew-Mandelstam SAID analysis of pion photoproduction data," Phys. Rev. C 86, 015202 (2012).

[168] R.L. Workman, W.J. Briscoe, M.W. Paris and I.I. Strakovsky, "Updated SAID analysis of pion photoproduction data," Phys. Rev. C 85, 025201 (2012).

[169] A.V. Anisovich, R. Beck, E. Klempt, V.A. Nikonov, A.V. Sarantsev, U. Thoma and Y. Wunderlich, "Study of ambiguities in $\pi^{-} p \rightarrow \Lambda K^{0}$ scattering amplitudes," Eur. Phys. J. A 49, 121 (2013).

[170] A.V. Anisovich et al., "Energy-independent PWA of the reaction $\gamma p \rightarrow K^{+} \Lambda$," Eur. Phys. J. A 50, 129 (2014).

[171] C. A. Paterson et al. [CLAS Collaboration], "Photoproduction of $\Lambda$ and $\Sigma^{0}$ hyperons using linearly polarized photons," Phys. Rev. C 93, 065201 (2016).

[172] A.V. Anisovich et al., "First (nearly) modelindependent confirmation of resonances in the fourth resonance region", in preparation.

[173] A. Svarc, M. Hadzimehmedovic, H. Osmanovic, J. Stahov, L. Tiator and R.L. Workman, "Introducing the Pietarinen expansion method into the singlechannel pole extraction problem," Phys. Rev. C 88, 035206 (2013).

[174] A. Svarc, M. Hadzimehmedovic, H. Osmanovic, J. Stahov, L. Tiator and R.L. Workman, "Pole positions and residues from pion photoproduction using the Laurent-Pietarinen expansion method," Phys. Rev. C 89, 065208 (2014).

[175] A. Svarc, M. Hadzimehmedovic, H. Osmanovic, J. Stahov, L. Tiator and R.L. Workman, "Generalization of the model-independent Laurent-Pietarinen singlechannel pole-extraction formalism to multiple channels," Phys. Lett. B 755, 452 (2016).

[176] K. M. Watson, "The Hypothesis of Charge Independence for Nuclear Phenomena," Phys. Rev. 85, 852 (1952). 